\title{
DELINEAMENTO EM ÂNGULOS
}

\author{
MARIA EUNICE O. C. RODRIGUES \\ Médica - Veterinária
}

Orientador: Prof. Dr. ANTONIO FRANCISCO IEMMA

\begin{abstract}
Dissertação apresentada à Escola Superior de Agricultura "Luiz de Queiroz", da Universidade de São Paulo, para obtenção do título de Mestre em Agronomia. Área de Concentração: Estatística $\theta$ Experimentação Agronômica.
\end{abstract}

PIRACICABA

Estado de São Paulo - Brasil

Julho - 1984 
Aos meus pais,

Ruy e Circe

\author{
Ao meu esposo \\ Abi 1 io \\ Ao meu filho \\ Rodrigo
}

DEDICO 


\section{AGRADEC I MENTOS}

Ao Dr. Antonio Francisco Iemma, Professor Assis tente Doutor do Departamento de Matemática e Estatística da ESALQ, pela orientação segura e de grande valia na realização deste trabalho.

Ao Dr. Humberto de Campos, Coordenador do Curso de Pós-Graduação em Estatística e Experimentação Agronômica, pela solicitude e incentivo que sempre nos proporcionou.

Ao Dr. Frederico Pimentel Gomes, pelo apoio, com preensão e ensinamentos desde o início de nossa caminhada.

Ao Colega Professor Lauro Boechat Batista, da Universidade Federal Rural do Rio de Janeiro, pelo valioso apoio na realização deste trabalho.

Ao Corpo Docente do Departamento de Matemática e Estatística da ESALQ, pelos conhecimentos que nos foram trans mitidos. 
Ao Dr. Jacintho Mendonça Jr. (in memoriam) pela confiança em nós depositada.

$\bar{A}$ Universidade Federal Fluminense pela oportuni dade do Curso.

A Srta. Maria Izalina Ferreira Alves pelo traba 1ho de datilografia.

A todos aqueles que de um modo ou de outro tenham colaborado para a conclusão deste trabalho. 
Í N D I C C E

Pàgina

RE SUMO

viii

S UMMARY

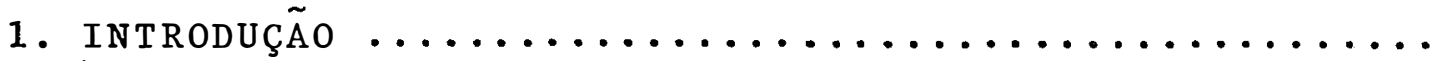

2. REvis Ão da Literatura $\ldots \ldots \ldots \ldots \ldots \ldots \ldots \ldots$

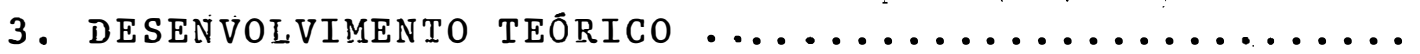

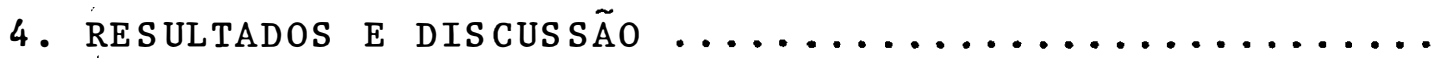

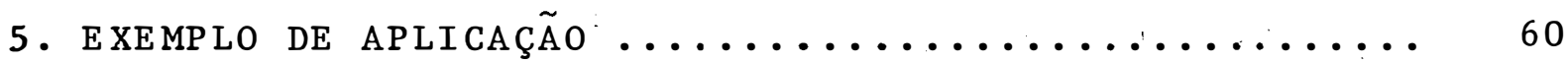

5.1. Determinação dos Coeficientes do Polinômio

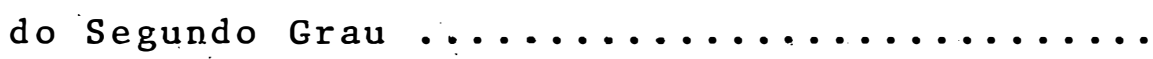

5.2. Determinação das Somas de Quadrados $\ldots \ldots \ldots \ldots$

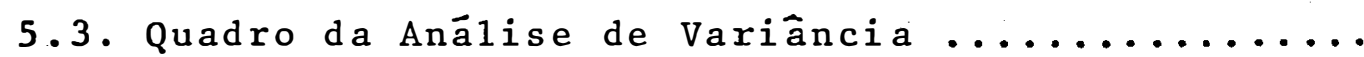


vi i

Pāgina

5.4. Determinação das Estimativas das Variâncias das Estimativas dos Coeficientes Polino-

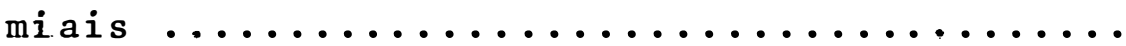

5.5. Determinação dos Valores de "t" para Testax as Hipóteses de que as Estimativas dos Coeficientes Polinomiais não Diferem de

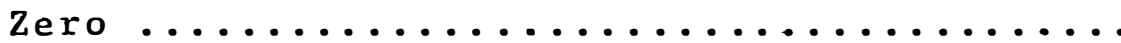

5.6. Determinação da Equação Polinomial ..........

5.7. Estudo do Máximo, Mínimo ou Ponto de Sela Através de Congruência de Matrizes ........

5.8. Determinação do Máximo da Função ............

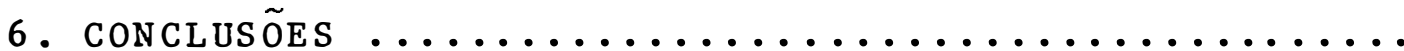

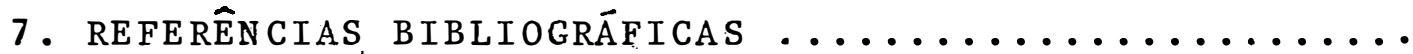




\title{
DELINEAMENTO EM ÂNGULOS
}

\author{
Autora: Maria Eunice O.C. Rodrigues
}

Orientador: Prof. Dr. Antonio Francisco Iemma

\section{RESUMO}

Foi apresentado um novo tipo de delineamento, denominado Delineamento em Ângulos; que tem por finalidade o. ajustamento aos dados, de um polinômio do segundo grau, com duas variáveis. Nesse delineamento, foram considerados oito novos pontos, em relação ao delineamento composto central, escolhidos de modo a ortogonalizar o referido delineamento.

o delineamento é formado por $16+$ p pontos, on de $P$ refere-se ao número de pontos centrais $(0,0)$, enquanto que 12 pontos dos demais referem-se a três fatoriais 2 x 2 nos quais os níveis, estão codificados em $-1 \mathrm{e}+1, \pm \delta$ cos $30^{\circ} \mathrm{e}$ $\pm \delta \operatorname{sen} 30^{\circ}, \pm \delta \cos 60^{\circ}$ e $\pm \delta$ sen $60^{\circ}$ e os quatro restan tes estão codificados em $(\delta, 0),(-\delta, 0),(0, \delta)$ e $(0,-\delta)$.

Foram obtidas as fórmulas que permitem a ortogo nalização do delineamento e as que determinam as estimativas 
dos coefichientes do polinômio do segundo grau com suas respectivas estimativas das variâncias, quando o delineamento é orto gonal, bem como suas respectivas somas de quadrados.

o Delineamento em Ângulos mostrou-se mais preciso que o Fatorial $3^{2}$ e menos preciso do que os Fatoriais $5^{2}$ e $7^{2}$ na estimação dos coeficientes polinomiais.

Levando em conta a mesma ärea total a ser gasta, o Delineamento em Ângulos é mais preciso quando se usa um ponto central e neste caso ele é mais preciso do que os fatoriais $5^{2}$ e $7^{2}$ na estimação dos coeficientes $\beta_{i}$ e $\beta_{I 2}$ e menos preciso que o fatorial $3^{2}, 5^{2}$ e $7^{2}$ na estimação do coeficiente $\beta_{i i}$. 


\title{
$A N G L E \cdot D E S I G N$
}

\author{
Author: Maria Eunice O.C. Rodrigues \\ Adviser: Antonio Francisco Iemma
}

\section{SUMMARY}

A new design was developed specifically for fitting to data a second-degree polynomial equation with two variable, denominated ANGLE DESIGN. Criteria in constructing the design were: to make it orthogonal when two factors are involved, and nine levels of each of the factors.

There were 16 treatment combinations and $P$ central points. Of the 16 treatment combinations, four from each of three $2^{2}$ factorial designs where the coded levels of the $x$-variable were -1 and $+1, \pm \delta$ sin $30^{\circ}$ and $\pm \delta \operatorname{cosin} 30^{\circ}$, and $\pm \delta \sin 60^{\circ}$ and $\pm \delta \operatorname{cosin} 60^{\circ}$, plus additional treatment combinations $(\delta, 0),(-\delta, 0),(0, \delta)$ and $(0,-\delta)$.

Formulas to make the design orthogonal, to estimate the polynomial equation coefficients, to estimate the variances of the polynomial regression coefficients, and to 
estimate the sums of square of the polynomial equation coefficients, were determined.

The Angle Design was more precise than the $3^{2}$ factorial design and less precise than the $5^{2}$ and $7^{2}$ factorial designs when we consider the estimation of the polynomial equation coefficients.

It was verified that the variances of the polynomial equation coefficients have a minimum when $P=1$, in the Angle Design, when it was used the same area.

When it was compared the precision of this design with one central point with the factorial desigrs, it was verified that it is more precise than the $5^{2}$, and $7^{2}$ factorial designs when it was considered the $\hat{\beta}_{i}$ and $\hat{\beta}_{12}$ coefficients. On the other hand, it was less precise than the $3^{2}, 5^{2}$, and $7^{2}$ fac torial designs, in the case of the $\hat{\beta}_{i i}$ coefficient. 


\section{INTRODUÇÃO}

Há quase meio século que os esquemas fatoriais começaram a ser estudados. Através desses esquemas, pode-se ajustar aos dados diversos modelos matemáticos com o intuito de se determinar os pontos criticos da superfície em estudo. Ẹmbora sejam notórias as vantagens dos esquemas fatoriais completos, foram introduzidos os fatoriais fracionārios de modo a diminuir o tamanho dos blocos quando há um núme ro grande de tratamentos.

$$
\text { Posteriormente, os compostos centrais foram }
$$

desenvolvidos com o objetivo de se ajustar aos dados polinômios do segundo grau e esse foi, certamente, um passo decisivo para o estudo de novos delineamentos.

Recentemente, vārios delineamentos vem sendo apresentados no estudo de superfícies de resposta, de modo a minimizar o custo de experimentação. 
o autor se propõe a apresentar um novo delineamento adequado ao estudo de superfície de resposta, com duas variäveis, onde existem 3 fatoriais $2^{2}$ acrescidos de pontos axiais, possibilitando aos pesquisadores a escolha dos valores de $P$ (pontos centrais) e $\delta$ (parâmetro) de modo a estimar os coeficientes polinomiais com melhor precisão'. 


\section{REVISÃO DA LITERATURA}

YATES (1937) introduziu na Estatistica Experimental os es quemas fatoriais, dos quais descreveu inúmeros tipos. Segundo o autor, quando hà um número elevado de tratamentos resultantes das combinações entre os niveis dos fatores, hä dificulḑades na eliminação de diferenças de fertilidą de dentro de uma mesma repetição e, consequentemente, o desvio padrão por par cela tende a ser alto em comparação com experimentos simples que envolvam pou cos tratamentos, podendo resultar dai uma pequena eficiência relativa dos es quemas fatoriais.

FINNEY (1945) apresentou os fatoriais fracionários com o objetivo de evitar c usc de um número grande de combinações entre os niveis dos fatores por bloco.

Os fatoriais fracionários foram estudados por diversos au tores, dentre eles DANIEL (1956), NELDER (1963) e MARGOLIN (1967).

DANIEL (1956) introduziu os conceitos de eficiēncia dos graus de liberdade e eficiência da variância para um fatorial fracionário.

A eficiēncia da variância de um fatorial fracionārio é ob tida. pela comparação da média das variāncias das estimativas dos éfeitos com a menor variância que poderia ser esperada com os fatores nos niveis \pm 1 . E 
a eficiència dos graus de liberdade é a razão entre nümero dos efeitos estimados e o nümero de graus de liberdade no delineamento. No estudo de eficiência dos graus de liberdade dos fatoriais fracionārios, o autor afirmou que as frações $2^{5-1}$ têm $100 \%$ de eficiência de graus de liberdade.

BOX e WILSON (1951) introduziram os delineamentos compostos centrais nos estudos de polinômios do segundo grau ajustados a dados provenientes destes delineamentos, com o intuito de minimizar o número de tratamentos.

o polinômio do segundo grau com $k$ variāveis, utilizados pelos autores, é dado pela expressão:

$$
Y_{u}=\beta_{0}+\stackrel{\aleph}{K}=1_{i} \beta_{i} x_{i u}+\sum_{i=1}^{K} \beta_{i i} x_{i u}^{2}+\sum_{i<j=2}^{K} \beta_{i j} x_{i u} x_{j u}+e_{u}
$$

Os delineamentos apresentados, chamados delinea mentos compostos, são construidos adicionando-se outros tratamentos ao fatorial $2^{k}$.

os delineamentos compostos são divididos em dois grupos, a saber: delineamentos compostos centrais (mais importantes) e delineamentos compostos não-centrais.

os delineamentos compostos centrais são obtidos adicionando ao esquema fatorial $2^{k}$ onde os níveis das variā- 
veis $x$ estão codificados em -1 e +1 , as seguintes combinações de tratamentos:

$$
\begin{aligned}
& (0,0, \ldots, 0) ;(-\alpha, 0, \ldots, 0) ; \quad(\alpha, 0, \ldots, 0) \\
& (0,-\alpha, \ldots, 0) ;(0, \alpha, \ldots, 0) \\
& \ldots \ldots \ldots \ldots \ldots \ldots \ldots \\
& (0,0, \ldots,-\alpha) ;(0,0, \ldots, \alpha)
\end{aligned}
$$

Assim, o número total de tratamentos é dado por $2^{k}+2 k+1$, sendo que no caso de $k=2$ resultam 9 trata mentos, como a seguir:

Delineamento Composto Central, com 2 fatores

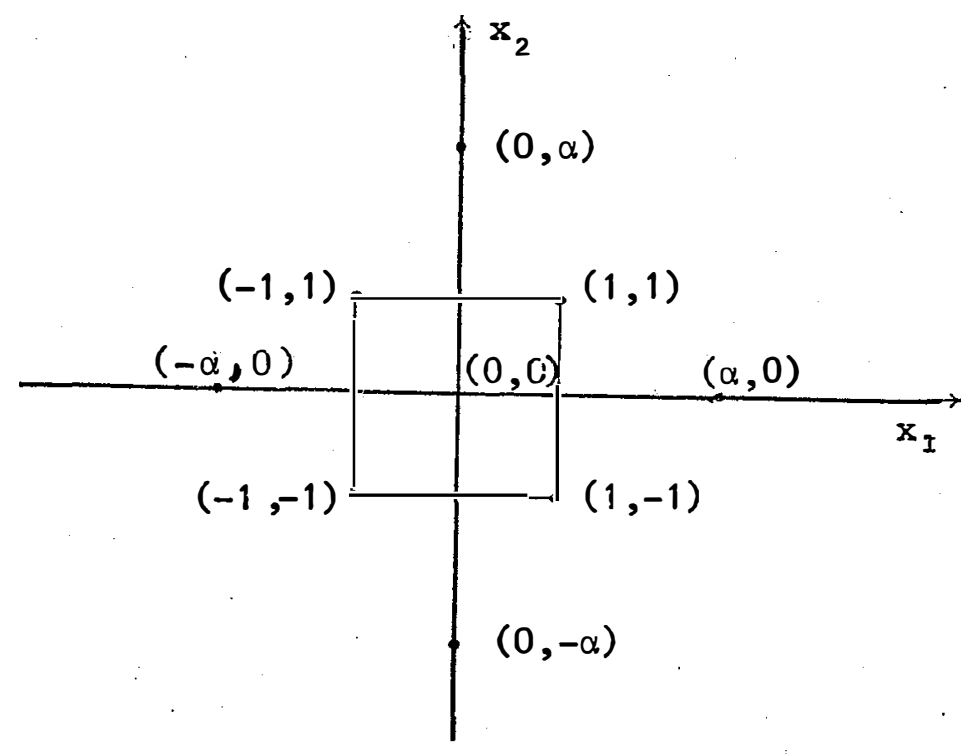


0 ponto central $(0,0)$ pode ser repetido $P$ vezes e nesse caso o delineamento fica constituído de $2^{k}+2 k+P$.

o valor de $\alpha$ pode ser escolhido para fazer os coeficientes do polinômio do segundo grau ortogonais uns aos outros, ou para minimizar os erros que são originados se averdadeira forma da superfície de resposta não é quadrática ou ainda para dar ao delineamento a propriedade de ser rotacional.

BOX e HUNTER (1957) propuseram o critério de rotacionalidade nos delineamentos compostos centrais, através do qual a variância da estimativa da resposta do polinômio de segundo grau é a mesma para todos os pontos equidistantes do ponto central. Os autores utilizaram o mesmo polinômio que BOX e WILSON (1951). No caso de dois fatores, o valor de $\alpha$ para que o composto central seja rotacional, é $\sqrt{2}$.

TRAMEL (1957), ROJAS $(1963,1972)$, STAVROU e CADY (1967) e VOSS e PESEK (1967) estudaram o assunto referente a superfície de resposta tendo sugerido diversos novos delineamentos.

TRAMEL (1957) sugeriu o delineamento "triplo cuㅡ bo", para três fatores, num total de 31 pontos, com nove níveis para cada fator, enquanto que STAVROU e CADY (1967) propu seram uma modificação no delineamento de TRAMEL (1957), colocando em dois blocos os tratamentos de cada cubo e repetindo 
os pontos axiais em cada bloco, com o objetivo de diminuir o erro experimental sem modificar a estimação dos parâmetros.

VOSS e PESEK (1967) propuseram um delineamento composto centra1 aumentado, com 19 pontos. $0 s 4$ tratamentos adicionais correspondem a meio cubo nos níveis \pm 2 .

ROJAS (1963) propôs o delineamento "San Cristóba1", delineamento este, originado do delineamento composto, onde foi incluido um tratamento testemunha. Em 1972 , ROJAS propôs o delineamento "San Cristóba1 Ortogonalizado".

No Brasil, os estudos mais aprofundados com re1 ação a superfície de resposta começaram com CAMPos (1967), que comparando o delineamento composto central rotativo para três fatores com um só ponto central e o fatorial $3^{3}$, concluiu que para ajustamento de superfície de resposta o fatorial é mais preciso do que o delineamento composto central rotativo.

Para chegar a esta conclusão, o autor se baseia nas variâncias das estimativas dos parâmetros, depois de reduzí-1as à mesma unidade, obtendo: 
Tabela 1. Variancias das estimativas dos parāmetros nos Delinea mentos Composto Central Rotativo e Fatorial $3^{3}$.

\begin{tabular}{lcc}
\hline PARÁmetros & $\begin{array}{c}\text { Delineamento Composto } \\
\text { Central }\end{array}$ & Rotativo \\
\hline Quadráticos & 0,7804 & 0,1667 \\
Interação & 0,6250 & 0,0833 \\
Lineares & 0,1190 & 0,0555 \\
\hline
\end{tabular}

PIMENTEL GOMES (1969) apresentou um estudo de determinação de ponto de máximo, ponto de minimo ou ponto de se 1a nas superfícies de resposta, usando o critērio de congruência de matrizes. Naquele trabalho, o autor informou que CAMPos (1967) encontrou $84 \%$ de casos de pontos de sela quando aplicou congruência de matrizes em 50 ensaios fatoriais de $3^{3}$, bem conduzidos, de adubação de milho $\operatorname{com} N, P$ e K.

PIMENTEL GOMES (1969) trabalhou com o mesmo modelo polinomial utilizado por BOX e WILSON (1951).

MORAES (1969), estudou, através de cälculos com putacionais, a superficie polinomial de um ensaio fatorial de adubação N, P, K, na Venezuela, em blocos casualizados, onde as doses foram não equidistantes. 
PENTEADO e BATISTA (1971) estudaram a eficiência do delineamento composto central em comparação com os fato riais completos de dois fatores e chegaram a conclusão de que o delineamento composto central é menos eficiente do que o fatorial $5 \times 5$.

PIMENTEL GOMES e CAMPOS (1972) propuseram correções de modo que os intervalos de comprimento fossem iguais quando estudaram a eficiência do delineamento composto central rotativo, com um ponto central, em relação ao fatorial $3^{3}$. Naquele trabalho, onde o intervalo entre os pontos $(-\sqrt{3}, 0,0)$ e $(\sqrt{3}, 0,0)$ é igual a $2 \sqrt{3}$, os autores propuseram correções de modo que o intervalo fosse igual ao do fatorial $3^{3}$ de comprimento 2. Para tanto, os autores multiplicaram as coor denadas dos pontos no delineamento composto central rotativo por $1 / \sqrt{3}$, obtendo as seguintes variâncias:

Tabela 2. Variāncias das estimativas dos parâmetros nos Delineamentos Compos to Central Rotativo e Fatorial $3^{3}$.

\begin{tabular}{lrrr}
\hline & Delineamento Composto & \\
& Central Rotativo & Fatorial $3^{3}$ \\
Quadráticos & 21,07 & $\sigma^{2}$ & \\
Interação & $16,88 \sigma^{2}$ & $4,50 \sigma^{2}$ \\
Lineares & $3,21 \sigma^{2}$ & $2,25 \sigma^{2}$ \\
\hline
\end{tabular}


BATISTA (1976) estudou a ortogonalização no delineamento composto central, com um máximo de quatro fatores e, determinou as fórmulas das variâncias das estimativas dos coeficientes do polinômio do segundo grau, com somente um ponto central. Nesse trabalho, o autor apresentou a seguinte ta bela que torna ortogonal o delineamento composto central:

Tabela 3. Valores de $\alpha$ que ortogonalizam o delineamento composto central, para $k$ variáveis.

\begin{tabular}{|l|ccc|}
\hline Valor de $k$ & 2 & 3 & 4 \\
\hline Valor de $\alpha$ & 1,000000 & 1,215412 & 1,414214 \\
\hline
\end{tabular}

\begin{abstract}
PENTEADO e BATISTA (1971), PIMENTEL GOMES e CAMPOS (1972), BATISTA (1976) e BATISTA e SilvA (1978) em seus estudos usaram o mesmo modelo matemātico que traduzia a equação polinomial utilizada por BoX e WILSON (1951).
\end{abstract}

BATISTA e SILVA (1978) estudaram também a ortogonalização do delineamento composto central, usando diversos pontos centrais, concluindo que quando são usados oito pontos centrais, a precisão da estimativa do coeficiente quadrático 
$B_{i i}$ é praticamente igual a precisão da estimativa do coeficiente 1 inear $\beta_{i}$.

BATISTA (1978) apresentou um novo tipo de delineamento, denominado "em círculos", que tem por base, na formu lação, os princípios de utilização de maior número de níveis para cada fator, menor número de combinações entre eles e inde pendência na estimação dos coeficientes. Consta de dois conjuntos de oito pontos com distâncias $\sqrt{2}$ e $\alpha \sqrt{2}$ do centro, e mais $P$ pontos centrais, que abrangem nove níveis diferentes de cada fator. Nesse trabalho o autor utilizou o mode 10 matemātico:

$$
\begin{aligned}
Y_{u}-\bar{Y}= & \beta_{1} X_{1 u}+\beta_{2} X_{2 u}+\beta_{11}\left(X_{1 u}^{2}-\overline{X_{1}^{2}}\right)+\beta_{22}\left(X_{2 u}^{2}-\overline{X_{2}^{2}}\right)+ \\
& +\beta_{12} X_{1 u} X_{2 u}+e_{u}
\end{aligned}
$$

que é semelhante ao proposto por BOX e WILSON (1951), onde com parando os dois modelos verifica-se que $\beta_{0}=\bar{Y}-\beta_{11} \bar{X}_{2}^{2}-\beta_{22} \bar{X}_{2}^{2}$ como vemos: 
a) $y_{u}=\beta_{0}+\beta_{1} x_{1 u}+\beta_{2} x_{2 u}+\beta_{11} x_{1 u}^{2}+\beta_{22} x_{2 u}^{2}+\beta_{12} x_{1 u} x_{2 u}+e_{u}$

b) $Y_{u}-\bar{Y}=\beta_{1} X_{1 u}+\beta_{2} x_{2 u}+\beta_{11}\left(x_{1 u}^{2}-\bar{x}_{1}^{2}\right)+\beta_{22}\left(x_{2 u}^{2}-\bar{X}_{2}^{2}\right)+$

$$
+\beta_{12} x_{1 u} X_{2 u}+e_{u}
$$

Igualando-se a à b tem-se:

$$
\beta_{0}=\bar{Y}-\beta_{11} \bar{X}_{1}^{2}-\beta_{22} \bar{X}_{2}^{2}
$$

CONAGIN (1979) estudou os delineamentos compostos centrais duplos que constam de dois conjuntos fatoriais, completos ou fracionários, nos níveis \pm 1 e \pm B, de um núme ro duplo de pontos axiais (nos níveis $\pm \alpha$ e \pm i $\alpha$ para cada um dos fatores) e por um certo número de pontos centrais. Os valores 1 e B representam os níveis da parte fatorial, enquanto que $\alpha$ e i $\alpha$ representam os níveis da parte axial. Tais delineamentos apresentam maior flexibilidade do que os compostos centrais pois permitem o estudo de $k$ fatores em vārios níveis ( 5 ou mais) e a partição ortogonal em blocos.

$$
\text { JORGE (1980) estudou o Delineamento Guadalupe }
$$
para três fatores, analisado através de modelo de regressão 
polinomial quadrática e concluiu que para ensaios de aduba ção, o Delineamento Guadalupe é menos eficiente que o fatorial $3^{3}$ e mais eficiente que o composto central origi na1. 


\section{DESENVOLVIMENTO TEORICO}

Suponha-se que se tenha uma idéia do ponto de máximo (ou de mínimo) da resposta do polinômio do segundo grau. Próximo deste ponto, fixa-se um ponto com coordenadas $(0,0)$, o qual poderá ser repetido P vezes. Em relação a este ponto, adiciona-se um fatorial $2 \times 2$ no qual os níveis estão codificados em -1 e +1 . A seguir são acrescentados mais dois fatoriais $2 \times 2$, sendo que no primeiro os níveis estão codifí cados em $\pm \delta \cos 30^{\circ}$ e $\pm \delta$ sen $30^{\circ}$ e no segundo os níveis es tão codificados em $\pm \delta \cos 60^{\circ}$ e $\pm \delta$ sen $60^{\circ}$. Finalmente adiciona-se os pontos codificados $(\delta, 0),(-\delta, 0),(0, \delta)$ e $(0,-\$)$. Assim, passa-se a ter um novo delineamento que será denominado Delineamento em Ângulos (figura 1).

Este novo delineamento poderā ter um máximo de 9 níveis para cada variável pois se, por exemplo, $\delta=1$, cada variável terá 7 níveis devido a coincidência dos níveis -1 e +1 do fatorial $2^{2}$ com os pontos axiais. 0 to 
Figura 1. Delineamento em Ângulos.

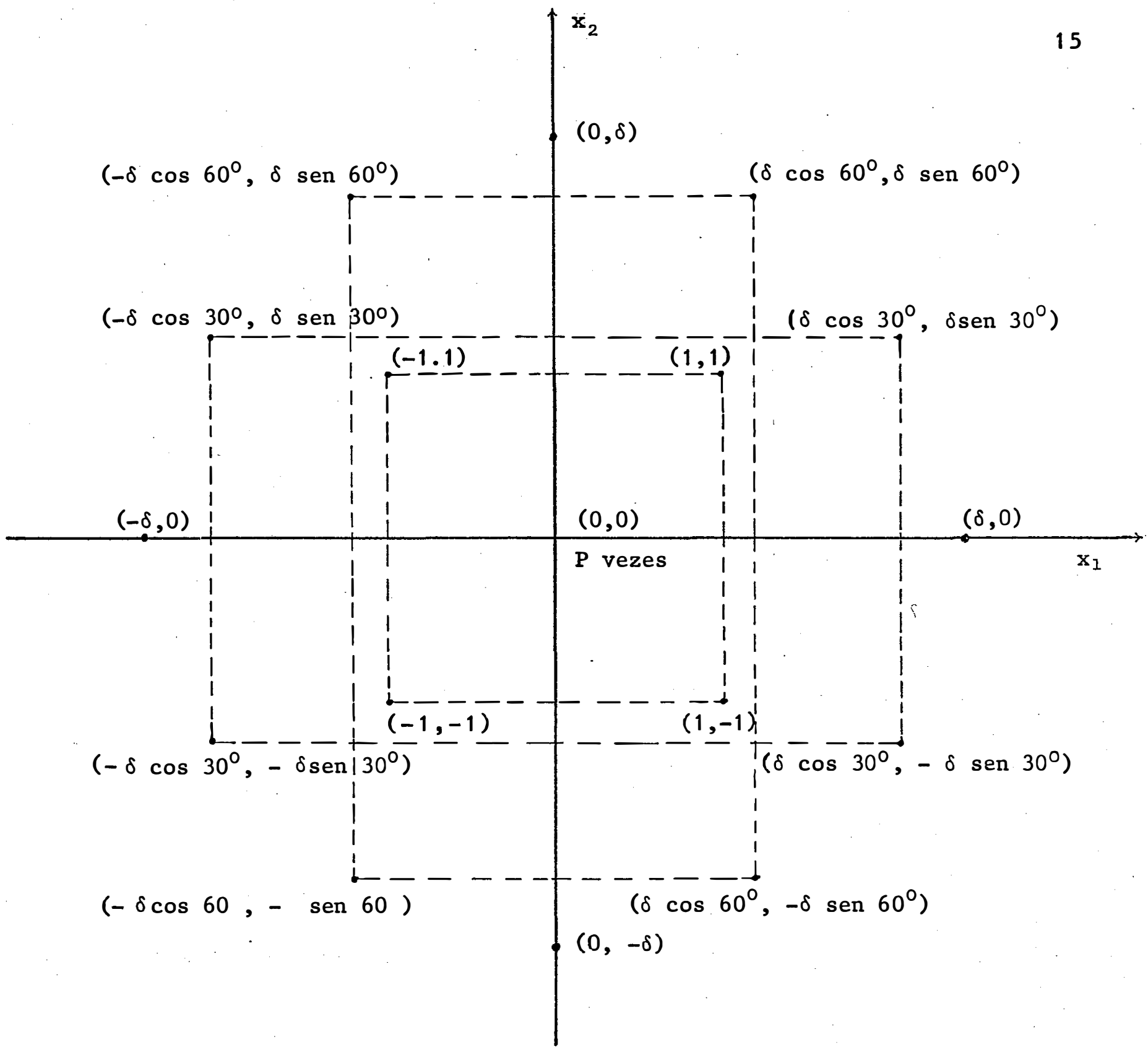


tal de números de combinações entre os níveis das variáveis seráa de $16+\mathbf{P}$.

o valor de $\delta$ poderá ser escolhido de modo a ortogonalizar o Delineamento em Ângulos. Para tanto, é neces sário que a matriz X'X obtida do modelo matemático seja diago na 1 .

3.1. Mode1o Matemático

0 modelo matemático de um polinómio do segundo grau que estima os coeficientes polinomiais neste delineamento è dado por:

$$
\begin{aligned}
y_{u}-\bar{Y}= & \beta_{1} X_{1 u}+\beta_{2} X_{2 u}+\beta_{11}\left(x_{1 u}^{2}-\overline{x_{1}^{2}}\right)+\beta_{22}\left(x_{2 u}^{2}-\overline{x_{2}^{2}}\right)+ \\
& +\beta_{12} x_{1 u} x_{2 u}+e_{u}
\end{aligned}
$$

A utilização desse modelo em detrimento do mode 1o usado por BOX e WILSON (1951), tem como objetivo a facilida de de cálculos, jā que ambos os modelos são semelhantes. Este modelo foi adotado pois simplifica a diagonalização da matriz X'X. 
Do modelo matemātico, temos em forma matricial, o seguinte sistema de equações:

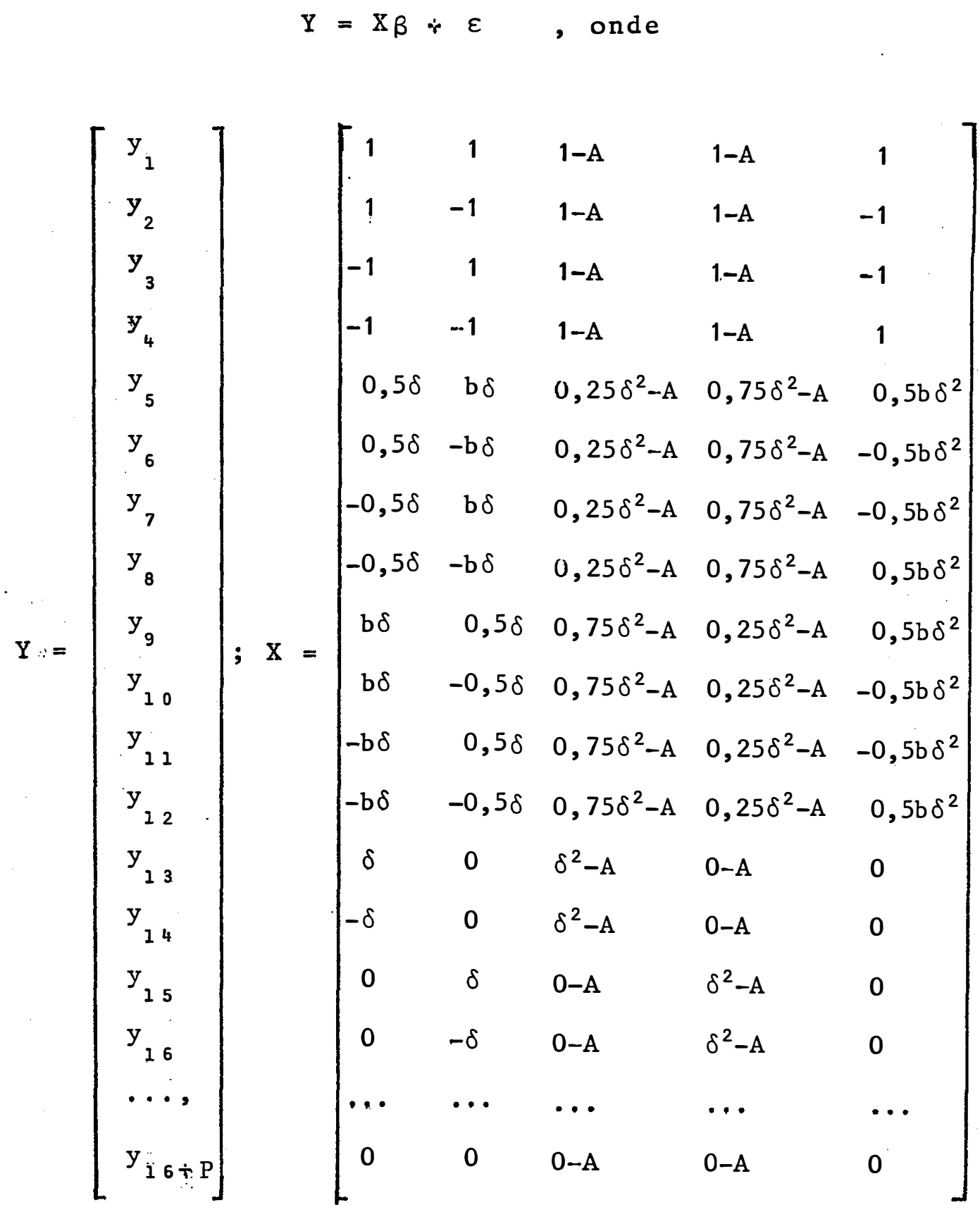




$$
\text { Onde: } \begin{aligned}
y_{u} & =Y_{u}-\bar{Y} \\
u & =1,2, \ldots, 16+P \\
\beta & =\left[\begin{array}{c}
\beta_{1} \\
\beta_{2} \\
\beta_{11} \\
\beta_{22} \\
\beta_{12}
\end{array}\right]
\end{aligned}
$$

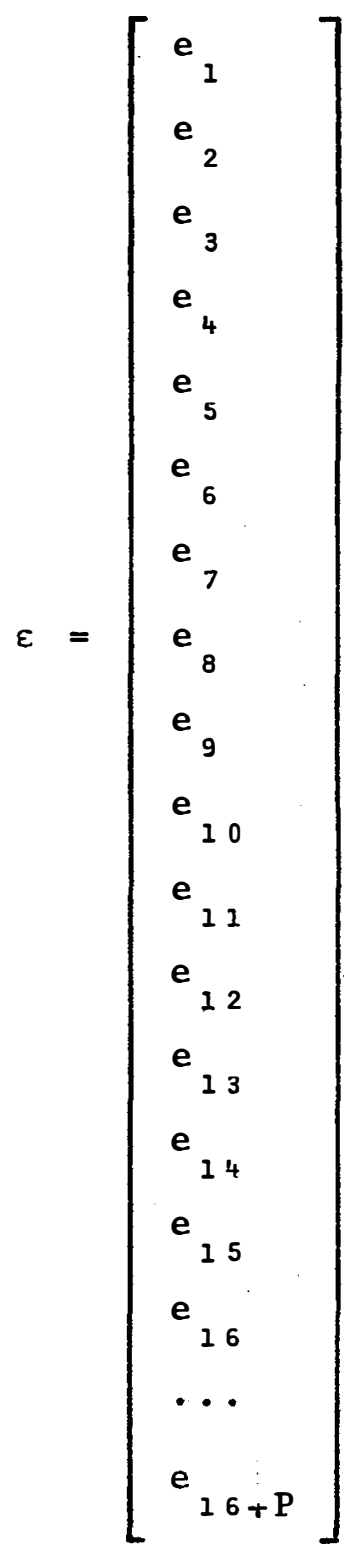


Sendo na matriz $X: 0,5=\operatorname{sen} 30^{\circ}=\cos 60^{\circ}$

$$
\begin{aligned}
& \mathrm{b}=\operatorname{sen} 60^{\circ}=\cos 30^{\circ}=0,8660254 \\
& \mathrm{~b}^{2}=0,75 \\
& \mathrm{~A}=\overline{\mathrm{X}_{1}^{2}}=\overline{\mathrm{X}_{2}^{2}}, 10 \mathrm{~g} 0
\end{aligned}
$$

$$
\begin{aligned}
A= & \frac{(1)^{2}+(1)^{2}+(-1)^{2}+(-1)^{2}+(0,5 \delta)^{2}+(0,5 \delta)^{2}+(-0,5 \delta)^{2}+(-0,5 \delta)^{2}+(b \delta)^{2}+(b \delta)^{2}+(-b \delta)^{2}+}{16+P} \\
& \quad \frac{\left(-b \delta j^{2}+(\delta)^{2}+(-\delta)^{2}+0^{2}+\ldots+0^{2}\right.}{16+P}
\end{aligned}
$$

$$
\begin{aligned}
A= & \frac{1+1+1+1 r 0,25 \delta^{2}+0,25 \delta^{2}+0,25 \delta^{2}+0,25 \delta^{2}+0,75 \delta^{2}+0,75 \delta^{2} T}{16+P} \\
& \frac{+0,75 \delta^{2}+0,75 \delta^{2} r \delta^{2} r \delta^{2}+0+\ldots+0}{16+P} \\
A= & \frac{4+4 \delta^{2}+2 \delta^{2}}{16+P} \quad \therefore \quad A=\frac{4 r 6 \delta^{2}}{16+P}
\end{aligned}
$$

3.2. Equações Normais

Através do método dos quadrados mínimos, que consiste em minimizar a soma dos quadrados dos erros, obtem-se as equações normais, como se segue: 


$$
\begin{aligned}
& Y=X \beta \dot{r} \varepsilon \\
& \varepsilon=Y-X \beta \\
& \varepsilon^{\prime}=\left(Y^{\prime}-\beta^{\prime} X^{\prime}\right)^{\prime} \\
& Z=\varepsilon^{\prime} \varepsilon=\left(Y^{\prime}-\beta^{\prime} X^{\prime}\right)(Y-X \beta) \\
& Z=Y^{\prime} Y-Y^{\prime} X \beta-\beta^{\prime} X^{\prime} Y r \beta^{\prime} X^{\prime} X \beta
\end{aligned}
$$

$$
\text { Mas } \beta^{\prime} X^{\prime} Y=Y^{\prime} X \beta \text {, pois ambas são escalares }
$$

Assim,

$$
\begin{aligned}
& z=Y^{\prime} Y-2 \beta^{\prime} X^{\prime} Y+\beta^{\prime} X^{\prime} X \beta \\
& d z=\Phi-2\left(d \beta^{\prime}\right) X^{\prime} Y+\beta^{\prime} X^{\prime} X(d \beta)+\left(d \beta^{\prime}\right) X^{\prime} X \beta
\end{aligned}
$$

$$
\text { Como } \beta^{\prime} x^{\prime} X(d \beta)=\left(d \beta^{\prime}\right) x^{\prime} X \beta \text {, pois ambas tem }
$$

dimensão 1 x 1 , vem:

$\mathrm{dz}=\Phi-2\left(\mathrm{~d} \beta^{\prime}\right) \mathrm{X}^{\prime} \mathrm{Y}-2\left(\mathrm{~d} \beta^{\prime}\right) \mathrm{X}^{\prime} \mathrm{X} \beta$

$d z=2 d \beta^{\prime}\left[X^{\prime} X \beta-X^{\prime} Y\right]$

As equações normais são obtidas quando iguala-se dz à $\Phi$. Portanto, vem:

$$
\begin{aligned}
& 2\left(\mathrm{~d}^{\prime}\right)\left[X^{\prime} X \hat{\beta}-X^{\prime} Y\right] \equiv \Phi \\
& \text { Assim, como d } \hat{\beta}^{\prime} \text { é sabidamente diferente de } \Phi
\end{aligned}
$$
pois é uma matriz de acrescimos, tem-se:

$$
X^{\prime} X \hat{\beta}-X^{\prime} Y=\Phi
$$


$X^{\prime} X \hat{\beta}=X^{\prime} Y$, que $\dot{e}$ o sistema de equações normais. Assim, facilmente pode-se obter a matriz $X^{\prime} X$, conforme descrito à seguir:

$$
X^{\prime} X=\left[\begin{array}{ccccc}
4+6 \delta^{2} & 0 & 0 & 0 & 0 \\
0 & 4+6 \delta^{2} & 0 & 0 & 0 \\
0 & 0 & D & M & 0 \\
0 & 0 & M & D & 0 \\
0 & 0 & 0 & 0 & 4+\left(3 / 2 \delta^{4}\right)
\end{array}\right]
$$

onde

$$
\begin{aligned}
& D=4+9 / 2 \delta^{4}-\frac{\left(4+6 \delta^{2}\right)^{2}}{16+P} \\
& M=4+3 / 2 \delta^{4}-\frac{\left(4+6 \delta^{2}\right)^{2}}{16+P}
\end{aligned}
$$

De fato, para D, tem-se:

$$
\begin{aligned}
D= & 4(1-A)^{2}+4\left(1 / 4 \delta^{2}-A\right)^{2}+4\left(3 / 4 \delta^{2}-A\right)^{2}+2\left(\delta^{2}-A\right)^{2}+(P+2)\left(0-A j^{2}\right. \\
D= & \left(4+4 A^{2}-8 A\right)+\left(1 / 4 \delta^{4}+4 A^{2}-2 A \delta^{2}\right)+\left(9 / 4 \delta^{4}+4 A^{2}-6 A \delta^{2}\right)+ \\
& +\left(2 \delta^{4}+2 A^{2}-4 A \delta^{2}\right)+(P+2) A^{2} \\
D= & 4+9 / 2 \delta^{4}+(P+16) A^{2}-A\left(8+12 \delta^{2}\right)
\end{aligned}
$$




$$
\begin{aligned}
& \mathrm{D}=4+9 / 2 \delta^{4}+\left[(\mathrm{P}+16) \mathrm{A}-\left(8+12 \delta^{2}\right)\right] \mathrm{A} \\
& \mathrm{D}=4+9 / 2 \delta^{4}+\left[(\mathrm{P}+16) \frac{\left(4+6 \delta^{2}\right)}{\mathrm{P}+16}-\left(8+12 \delta^{2}\right)\right] \mathrm{A} \\
& \mathrm{D}=4+9 / 2 \delta^{4}+\left[4+6 \delta^{2}-8-12 \delta^{2}\right] \mathrm{A} \\
& \mathrm{D}=4+9 / 2 \delta^{4}+\left[-4-6 \delta^{2}\right] \mathrm{A} \\
& \mathrm{D}=4+9 / 2 \delta^{4}-\left[4+6 \delta^{2}\right] \mathrm{A} \\
& \mathrm{D}=4+9 / 2 \delta^{4}-\left[4+6 \delta^{2}\right] \frac{\left[4+6 \delta^{2}\right]}{16+P} \\
& D=4+9 / 2 \delta^{4}-\frac{\left(4+6 \delta^{2}\right)^{2}}{16+P}
\end{aligned}
$$

De modo anälogo, para M, vem:$$
M=4(1-A)^{2}+8\left(1 / 4 \delta^{2}-A\right)\left(3 / 4 \delta^{2}-A\right)+4\left(\delta^{2}-A\right)(0-A)+P(0-A)^{2}
$$$$
M=4+3 / 2 \delta^{4}+A^{2}(P+16)-A\left(8+12 \delta^{2}\right)
$$$$
M=4+3 / 2 \delta^{4}+A\left[(P+16) A-\left(8+12 \delta^{2}\right)\right]
$$$$
M=4+3 / 2 \delta^{4}+A\left[(P+16) \frac{\left(4+6 \delta^{2}\right)}{16+P}-\left(8+12 \delta^{2}\right)\right]
$$$$
M=4+3 / 2 \delta^{4}+\mathrm{A}\left[4+6 \delta^{2}-8-12 \delta^{2}\right]
$$$$
M=4+3 / 2 \delta^{4}+A\left(-4-6 \delta^{2}\right)
$$$$
M=4+3 / 2 \delta^{4}-A\left(4+6 \delta^{2}\right)
$$$$
M=4+3 / 2 \delta^{4}-\frac{\left(4+6 \delta^{2}\right)}{16+P}\left(4+6 \delta^{2}\right)
$$ 


$$
M=4+3 / 2 \delta^{4}-\frac{\left(4+6 \delta^{2}\right)^{2}}{16+P}
$$

E a matriz X'Y é dada por:

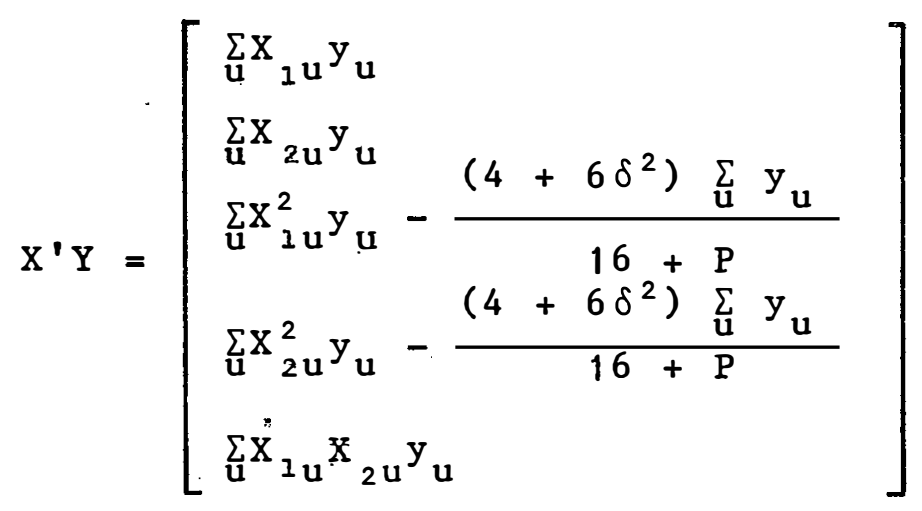

Assim, o sistema de equações normais tem a for-

ma:

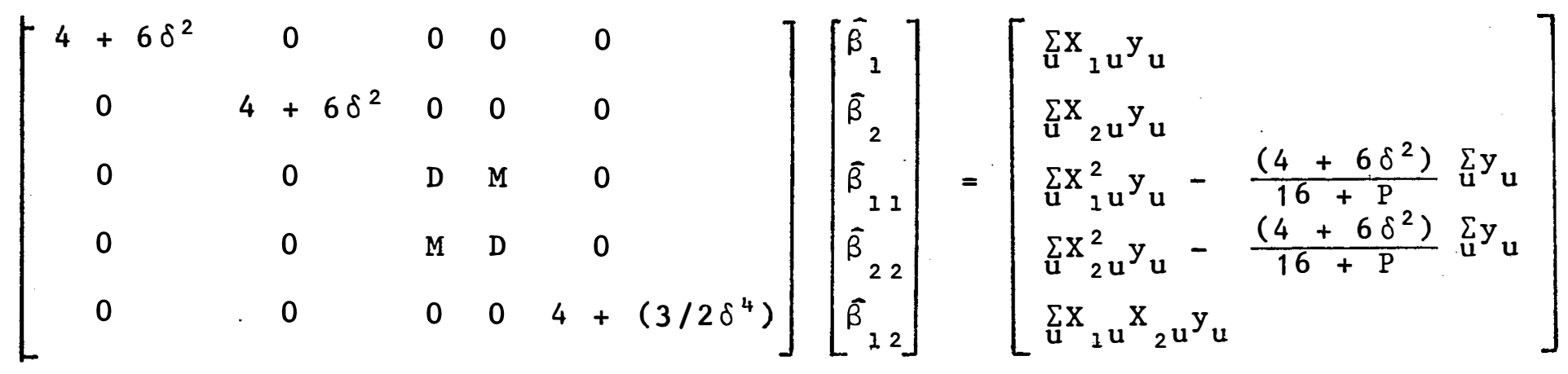


onde:

$$
\begin{aligned}
& M=4+3 / 2 \delta^{4}-\frac{\left(4+6 \delta^{2}\right)^{2}}{16+P} \\
& D=4+9 / 2 \delta^{4}-\frac{\left(4+6 \delta^{2}\right)^{2}}{16+P}
\end{aligned}
$$

3.3. Solução do Sistema

Das equaçộes normais $X^{\prime} X \hat{\beta}=X^{\prime} Y$, obtem-se a solução do sistema através da matriz inversa ( $\left.X^{\prime} X\right)^{-1}$, pois a matris X'X é não singular. Portanto, vem:

$$
\begin{aligned}
& X^{\prime} X \hat{\beta}=X^{\prime} Y \\
& \left(X^{\prime} X\right)^{-1}\left(X^{\prime} X\right) \hat{\beta}=\left(X^{\prime} X\right)^{-1} X^{\prime} Y \\
& I \hat{\beta}=\left(X^{\prime} X\right)^{-1} X^{\prime} Y \\
& \hat{\beta}=\left(X^{\prime} X\right)^{-1} X^{\prime} Y
\end{aligned}
$$




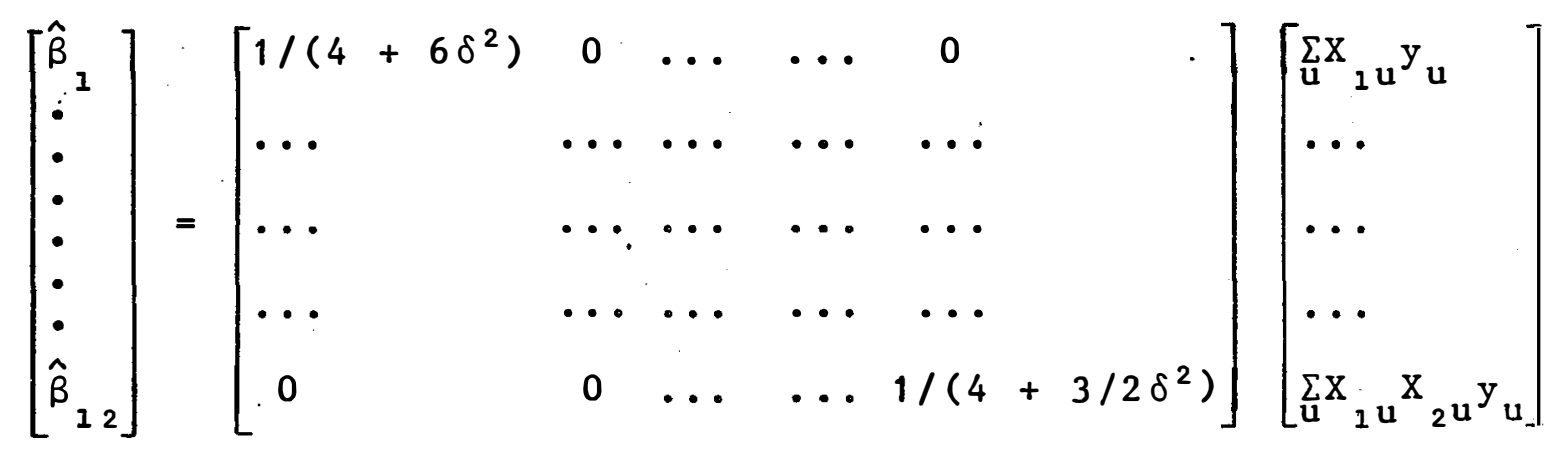

Por exemplo, para $\hat{\beta}_{1}$ e fazendo $M=0$, vem:

$\hat{\beta}_{1}=\frac{1}{4+6 \delta^{2}} \cdot \sum_{u} x_{1} u^{y}$

e de modo análogo para os demais coeficientes polinomiais.

3.4. Soma de Quadrados

A soma dos quadrados devido aos erros é dada

por:

$$
\begin{aligned}
& \text { SQ erro }=\hat{\varepsilon}^{\prime} \hat{\varepsilon} \\
& \text { SQ erro }=\left(Y^{\prime}-\hat{\beta}^{\prime} X^{\prime}\right)(Y-X \hat{\beta}) \\
& \text { SQ erro }=Y^{\prime} Y-Y^{\prime} X \hat{\beta}-\hat{\beta}^{\prime} X^{\prime} Y+\hat{\beta}^{\prime} X^{\prime} X \hat{\beta} \\
& \text { Como } X^{\prime} X \hat{\beta}=X^{\prime} Y, \text { vem: } \\
& \text { SQ erro }=Y^{\prime} Y-Y^{\prime} X \hat{\beta}-\hat{\beta}^{\prime} X^{\prime} Y+\hat{\beta}^{\prime} X^{\prime} Y \\
& \text { SQ erro }=Y^{\prime} Y-Y^{\prime} X \hat{\beta}-\Phi
\end{aligned}
$$


Como $Y^{\prime} X \hat{\beta}=\hat{\beta}^{\prime} X^{\prime} Y$, pois $Y^{\prime} X \bar{\beta}$ é uma matriz $1 \times 1$,

vem :

$S Q$ erro $=Y^{\prime} Y-\hat{\beta}^{\prime} X^{\prime} Y$

Assim, a soma dos quadrados devido aos parâme-

tros é dada por:

$$
\begin{aligned}
& \text { SQ Par }=\hat{\beta}^{\prime} X^{\prime} Y \text {, onde } \\
& \hat{\beta}^{\prime}=\left[\begin{array}{lllll}
\hat{\beta}_{1} & \hat{\beta}_{2} & \hat{\beta}_{11} & \hat{\beta}_{22} & \hat{\beta}_{12}
\end{array}\right] \quad e
\end{aligned}
$$

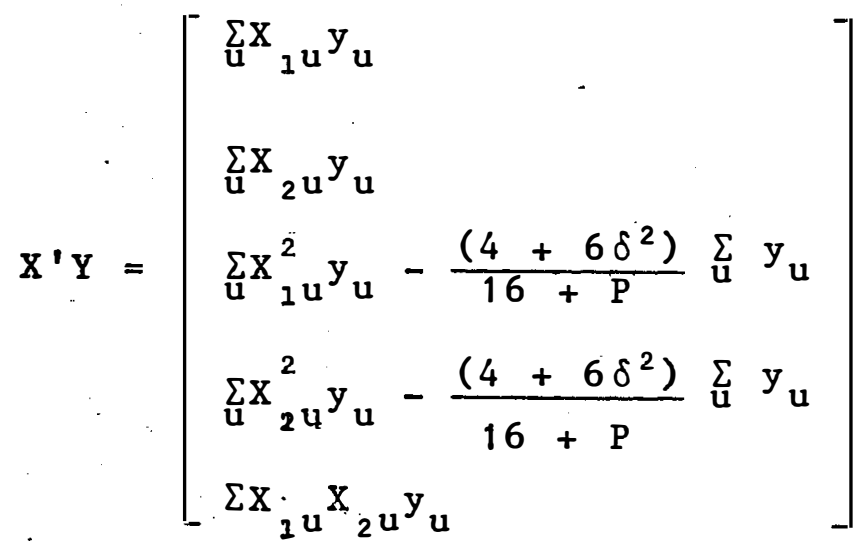

3.5. Variāncias das estimativas

As variâncias das estimativas dos coeficientes polinomiais serão dadas atravēs da matriz de dispersão (matriz de variāncias e covariâncias) que è obtida, segundo SEARLE (1971), dentre outros, por:

$$
V(\bar{\beta})=E[\bar{\beta}-E(\hat{\beta})][\bar{\beta}-E(\hat{\beta})]^{\prime}
$$




$$
\begin{aligned}
& V(\hat{\beta})=E\left\{\left(X^{\prime} X\right)^{-1} X^{\prime} Y-E\left[\left(X^{\prime} X\right)^{-1} X^{\prime} Y\right]\right\}\left\{\left(X^{\prime} X\right)^{-1} X^{\prime} Y-E\left[\left(X^{\prime} X\right)^{-1} X^{\prime} Y\right]\right\}^{\prime} \\
& V(\hat{\beta})=E\left(X^{\prime} X\right)^{-1} X^{\prime}[Y-E(Y)]\left[Y^{\prime}-E\left(Y^{\prime}\right)\right] X\left(X^{\prime} X\right)^{-1} \\
& V(\hat{\beta})=\left(X^{\prime} X\right)^{-1} X^{\prime} E\left(\varepsilon \varepsilon^{\prime}\right) X\left(X^{\prime} X\right)^{-1} \\
& M a s E\left(\varepsilon \varepsilon^{\prime}\right)=\sigma^{2}, 10 g 0: \\
& V(\hat{\beta})=\left(X^{\prime} X\right)^{-1} X^{\prime} X\left(X^{\prime} X\right)^{-1} \sigma^{2} \\
& V(\hat{\beta})=\left(X^{\prime} X\right)^{-1} I \sigma^{2} \\
& V(\hat{\beta})=\left(X^{\prime} X\right)^{-1} \sigma^{2}
\end{aligned}
$$

3.6. Testes de significância

0 valor de "t" para testar a hipótese $H_{0}: \beta_{i}=$

$=0$, é dado por:

$$
t=\frac{\hat{\beta}_{i}}{\sqrt{ } V\left(\hat{\beta}_{i}\right)} \text {, onde } V\left(\hat{\beta}_{i}\right) \text { é obtida como em } 3.5
$$

3.7. Estudo do máximo, minimo ou ponto de sela através de congruência de matrizes

$$
\text { Se a matriz } B=\left[\begin{array}{ll}
\hat{\beta} & \hat{\beta}_{12}^{12} \\
\hat{\beta}_{12} & \hat{\beta}_{22}
\end{array}\right] \text { for definida positi }
$$

va então haverá ponto de minimo e se for definida negativa então haverá ponto de máximo. 
0 método consiste em transformar a matriz B numa matriz diagonal por meio de operaçöes de congruência conforme descrito em PIMENTEL GOMES (1969), entre outros. Se na matriz diagonal os termos da diagonal principal forem maiores do que zero a matriz será definida positiva; enquanto que se os ter mos forem menores do que zero a matriz será definida negativa. Se, no entanto, houver termos positivos e negativos, ela será não definida.

Assim, por exemplo, para a matriz B dada a seguir.

$$
\begin{aligned}
& B=\left[\begin{array}{rr}
-5 & 2 \\
2 & -4
\end{array}\right] \\
& \mathrm{L}_{2}=5 \mathrm{~L}_{2}+2 \mathrm{~L}_{1} \\
& B=\left[\begin{array}{rr}
-5 & 2 \\
0 & -16
\end{array}\right] \\
& C_{2}=5 \mathrm{C}_{2}+2 \mathrm{C}_{1} \\
& B=\left[\begin{array}{rr}
-5 & 0 \\
0 & -80
\end{array}\right]
\end{aligned}
$$

onde L e C são linhas e colunas.

Como a matriz B é definida negativa, então a fun cão atinge ponto de máximo. 


\section{RESULTADOS E DISCUSSÃO}

$$
\text { Procurando ajustar aos dados do Delineamento }
$$
em Ângulos o modelo matemático dado por:

$$
\begin{aligned}
Y_{u}-\bar{Y}= & \beta_{1} x_{1 u}+\beta_{2} x_{2 u}+\beta_{11}\left(x_{1 u}^{2}-\overline{x_{1}^{2}}\right)+\beta_{22}\left(x_{2 u}^{2}-\overline{x_{2}^{2}}\right)+ \\
& +\beta_{12} x_{1 u} x_{2 u}+e_{u}
\end{aligned}
$$

ter-se-á, primeiramente, em forma matricial a matriz $x$.

As estimativas dos coeficientes do polinômio do segundo grau (e.1) serão independentes, se a matriz X'X for diagonal. Assim, para tornar ortogonal o Delineamento em Ângulos deve-se diagonalizar $X^{\prime} X$, isto é, deve-se igualar Ma zero $(M=0)$. Portanto vem: 


$$
4+3 / 2 \delta^{4}-\frac{\left(4+6 \delta^{2}\right)^{2}}{16+P}=0 \quad(\mathrm{e} \cdot 2)
$$

Desenvolvendo, tem-se:

$$
(3 / 2 P-12) \delta^{4}-48 \delta^{2}+4(12+P)=0 \quad(e .3)
$$

Analisando a expressão 3 :

a) Se $P=8$, então

$$
\begin{gathered}
(3 / 2 \cdot 8-12) \delta^{4}-48 \delta^{2}+4(12+8)=0 \\
-48 \delta^{2}+80=0 \\
\delta^{2}=5 / 3 \text { e, portanto, } \delta=1,290994 \text { (e.4) }
\end{gathered}
$$

b) Se $1 \leq \mathrm{P}<8$, neste caso tem-se:

$$
(3 / 2 P-12) \delta^{4}-48 \delta^{2}+4(12+P)=0
$$

Como $1 \leq \mathrm{P}<8$, implica dizer que $(3 / 2 \mathrm{P}-12)$ é negativo. Portanto, para se resolver a equação deve-se mul tiplicar por $(-1)$, fazendo; 


$$
(12-3 / 2 P) \delta^{4}+48 \delta^{2}-4(12+P)=0
$$

e assim obtem-se:

$$
\begin{aligned}
& \delta^{2}=\frac{-48 \pm \sqrt{48^{2}+4(12-3 / 2 P)(12+P) 4}}{2(12-3 / 2 P)} \\
& \delta^{2}=\frac{-24 \pm \sqrt{1152-24 P-6 P^{2}}}{(12-3 / 2 P)}
\end{aligned}
$$$$
\delta=\left(\frac{-24 \pm \sqrt{1152-24 P-6 P}}{(12-3 / 2 P)}\right)^{1 / 2}
$$

c) Se $8<P \leq 12$, então:

$$
(3 / 2 P-12) \delta^{4}-48 \delta^{2}+4(12+P)=0
$$

Resolvendo, tem-se: 


$$
\delta^{2}=\frac{48 \pm \sqrt{48^{2}-4(3 / 2 P-12)(12+P) 4}}{2(3 / 2 P-12)}
$$

$$
\delta^{2}=\frac{24 \pm \sqrt{1152-24 P-6 P^{2-}}}{(3 / 2 P-12)}
$$

$$
\delta=\left(\frac{24 \pm \sqrt{1152-24 \mathrm{P}-6 \mathrm{P}^{z-1}}}{(3 / 2 \mathrm{P}-12)}\right)^{1 / 2}
$$

Na expressão 6 pode-se facilmente verificar que o máximo valor assumido por $P$ é 12 , caso contrārio haveria raiz quadrada de um número negativo.

Pode-se notar pelas três fórmulas de $\delta$, que tor nam ortogonal o Delineamento em Ângulos, que existem dois valo res de $\delta$ para cada valor de $P$, quando $P$ está compreendido no intervalo de 9 a 11 , pois neste caso, como se vê na expressão 6 há duas raízes positivas que satisfazem a condição de ortogonalização.

Atribuindo valores a $P$ de 1 à 12 , obtem-se uma tabela na qual estão os lvalores de $\delta$ que tornam ortogonal o Delinea mento em Ângulos com $P$ pontos centrais (tabela 1 ). 
Por exemplo, quando hà somente um ponto central $(P=1)$, o valor de $\delta$ que torna ortogonal o Delineamento em Ângulos será:

$$
\begin{gathered}
\delta=\left(\frac{-24 \pm \sqrt{1152-24-6}}{(12-3 / 2)}\right)^{1 / 2} \\
\delta=0,9510029
\end{gathered}
$$

A outra raiz é de nümero negativo e não interes sa.

Pode-se verificar pela Tabela 4 e Figura 2 que quando se usam 1 ou 2 pontos centrais, os quatro pontos extremos do Delineamento em Angulos são os correspondentes ao Fatorial $2 \times 2$, no qual os niveis estão codificados em -1 e +1 , pois $\delta$, nesses casos, é menor do que 1 . 
Tabela 4. Valores de $\delta$ que tornam ortogonal

o Delineamento em Ângulos, obtidos através das expressões 4,5 e 6 .

\begin{tabular}{cc}
\hline Valor de $P$ & Valor de $\delta$ \\
\hline 1 & 0,951003 \\
3 & 0,992380 \\
4 & 1,034810 \\
5 & 1,078878 \\
6 & 1,125313 \\
7 & 1,175088 \\
8 & 1,229594 \\
9 & 1,290994 \\
9 & 1,363035 \\
10 & 5,490185 \\
10 & 1,453327 \\
11 & 3,726639 \\
11 & 1,582690 \\
12 & 2,856880 \\
\hline 100000 \\
\hline 10
\end{tabular}


Figura 2. Delineamento em Ângulos, com $P \leq 2$.

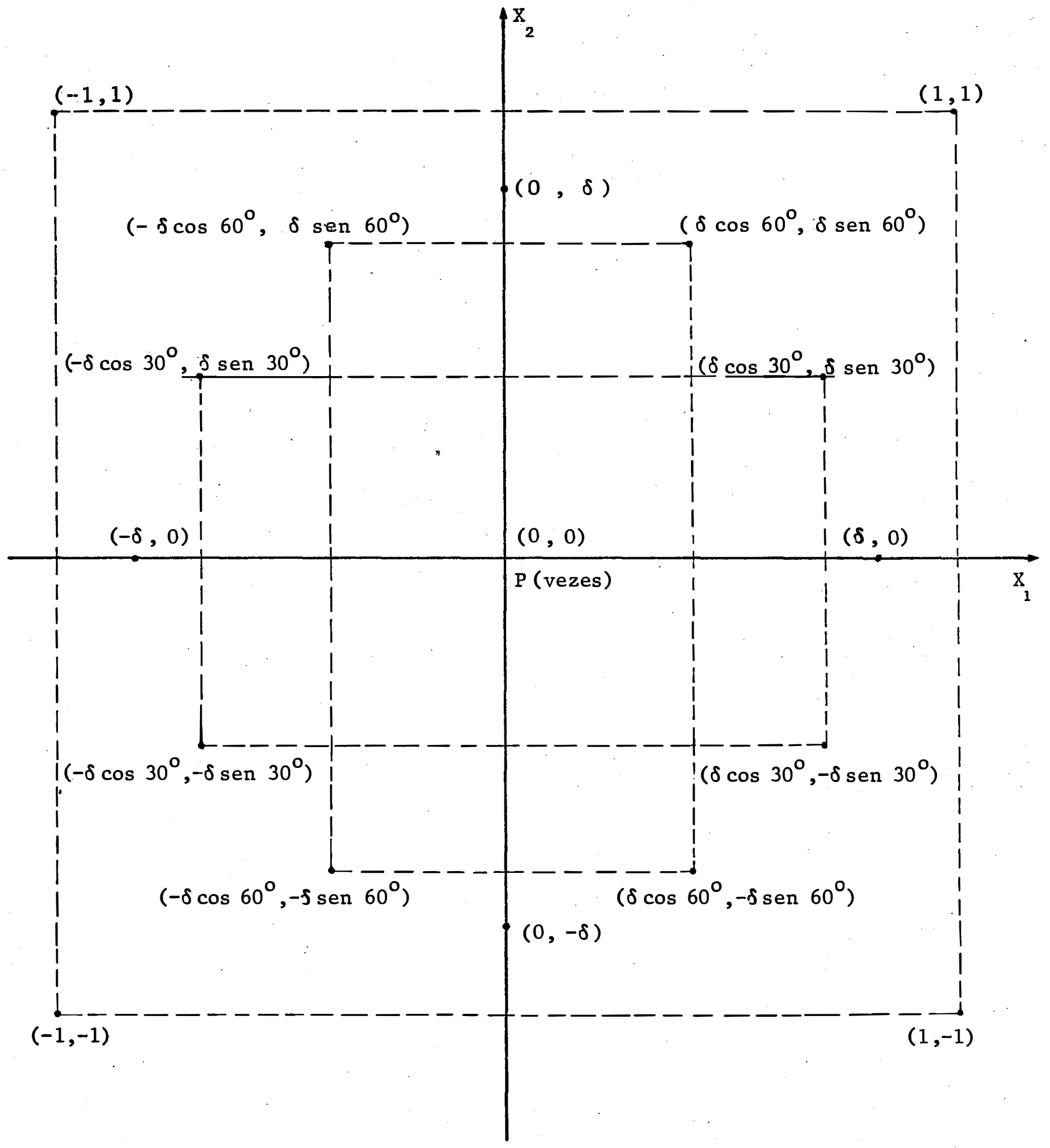




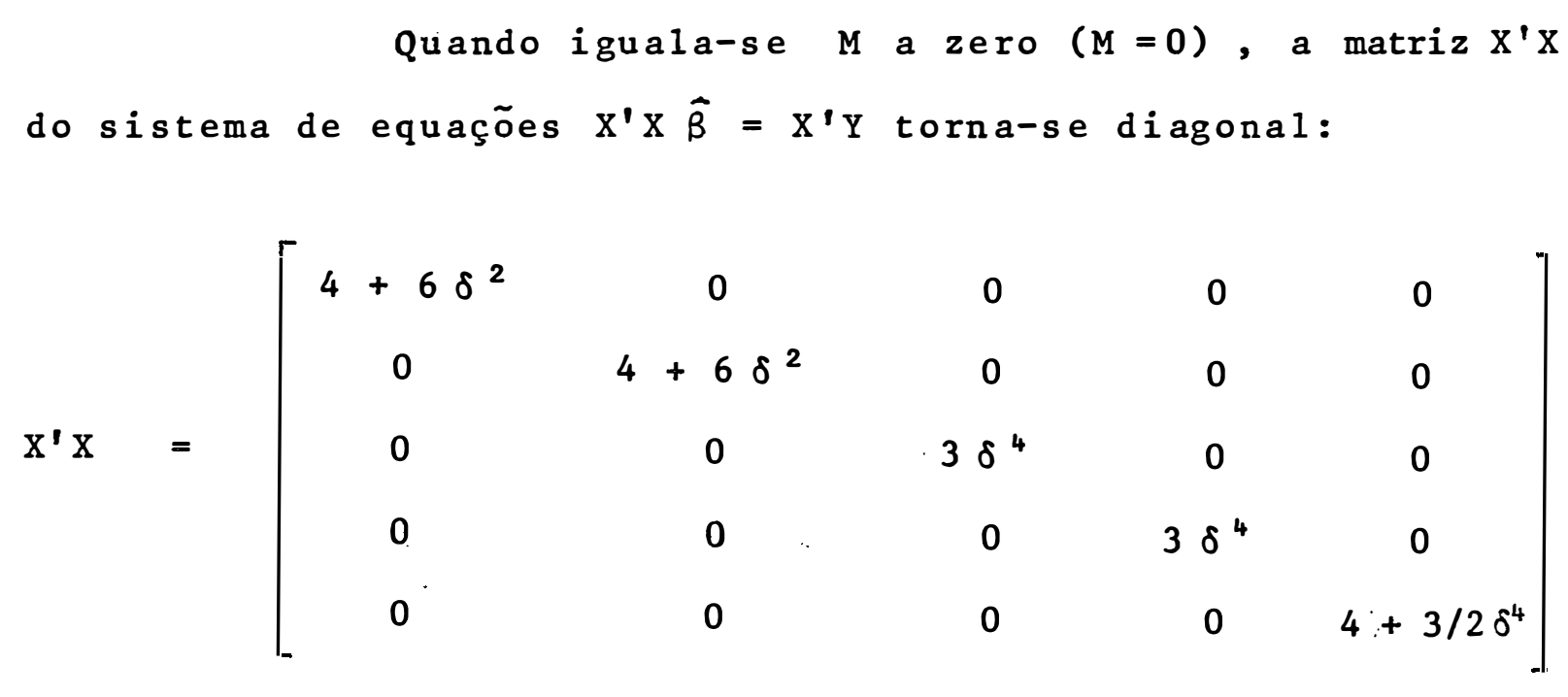

Assim sua inversa é facilmente obtida.

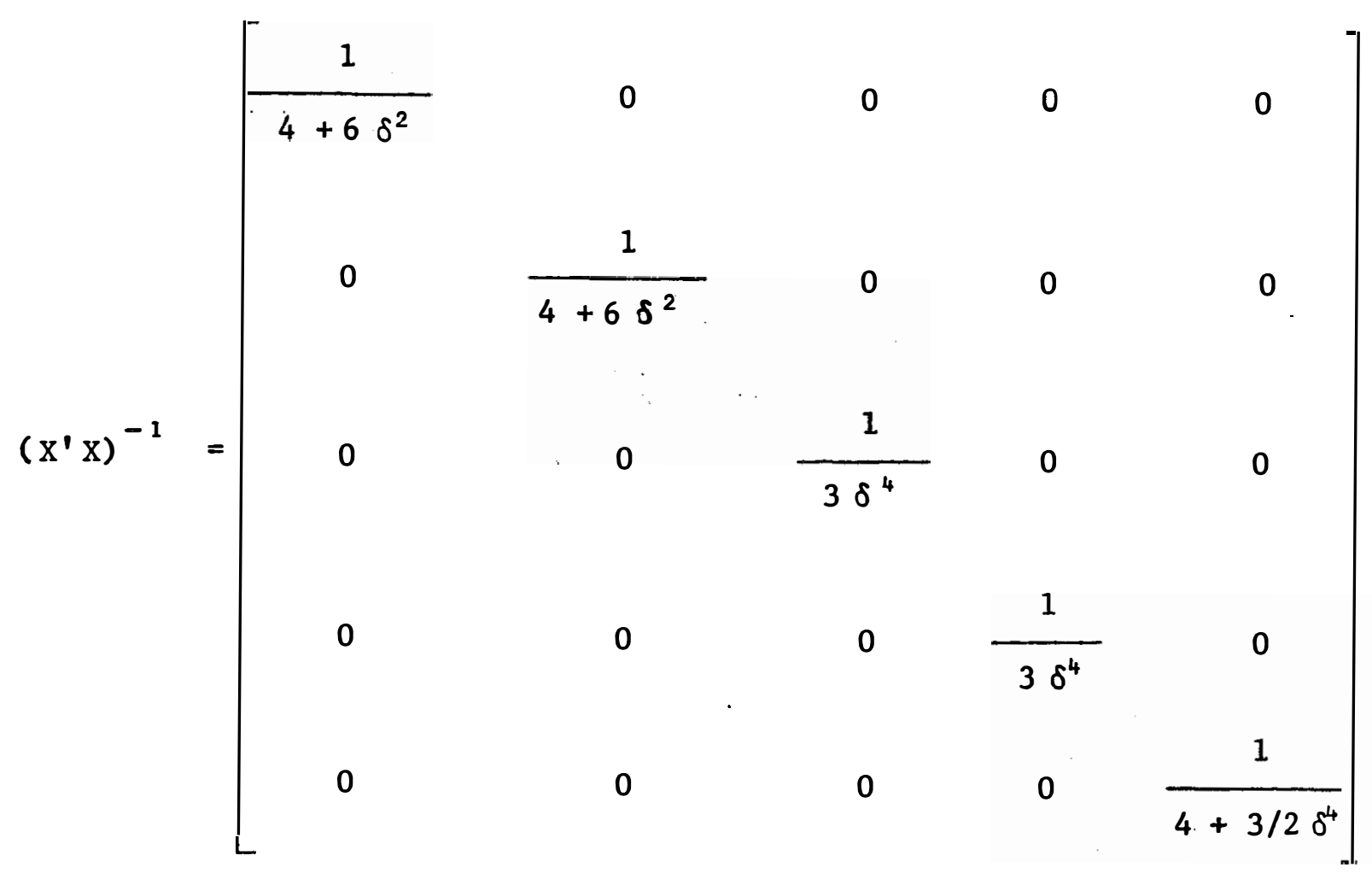


Entretanto, os cálculos foram efetuados para uma repetição e para $\underline{r}$ repetições os elementos da matriz $X^{\prime} X$ ficarão multiplicados por $\underline{r} e$, consequentemente, os elemen tos da inversa da matriz diagonal X'X ficarão divididos por $\underline{r}$ :

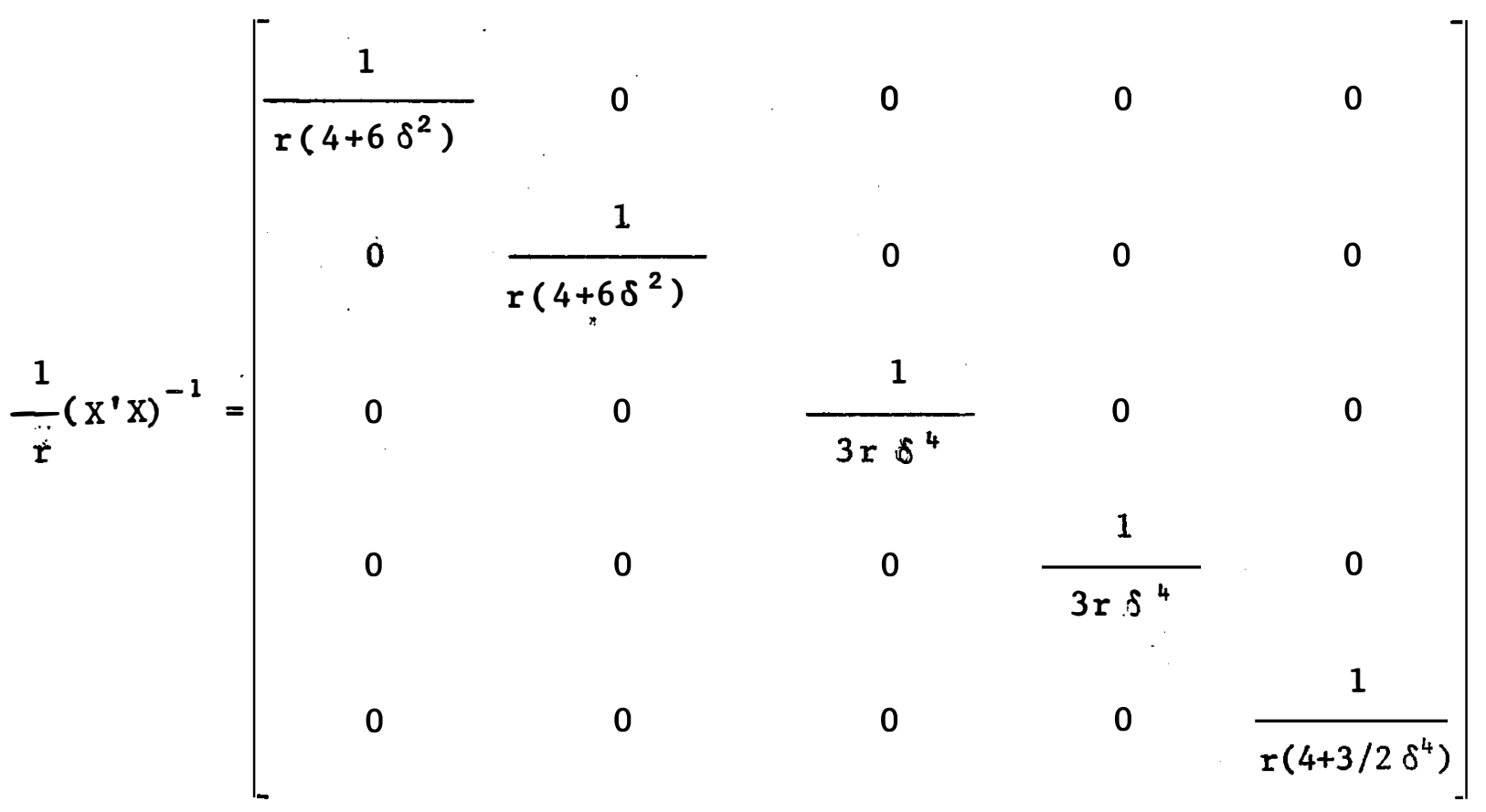

Pode-se assim notar que os elementos correspondentes aos coeficientes $\hat{\beta}_{i}, \hat{\beta}_{i i}$ e $\hat{\beta}_{-12}$ são respectivamente:

$$
\frac{1}{r\left(4+6 \delta^{2}\right)}=\frac{1}{3 r \delta^{4}} \text { e } \frac{1}{r\left(4+3 / 2 \delta^{4}\right)}
$$


Então, segundo o item 3.5 , tem-se:

$$
\begin{aligned}
& \hat{v}\left(\hat{\beta}_{i}\right)=\frac{1}{r\left(4+6 \delta^{2}\right)} \cdot \hat{\sigma}^{2}, \hat{v}\left(\hat{\beta}_{i i}\right)=\frac{1}{3 r \delta^{4}} \cdot \hat{\sigma}^{2} e \\
& \hat{V}\left(\hat{\beta}_{12}\right)=\frac{1}{r\left(4+3 / 2 \delta^{4}\right)} \cdot \hat{\sigma}^{2}
\end{aligned}
$$

Nota-se pelas fórmulas, que à medida que o valor de $\delta$ aumenta as estimativas das variâncias diminuem.

$$
\text { Atribuindo valores a } P \text { de } 1 \text { a } 12 \text {, tem-se a tabe }
$$
1a das estimativas das variâncias das estimativas dos coeficien tes do polinómio do segundo grau, com duas variáveis, no Delineamento em Ângulos (Tabela 5). 
Tabela 5. As estimativas das variâncias das estimativas dos coeficientes do polinômio de segundo grau, com dois fatores, quando o Delineamento em Ângulos é ortogonal. Os valores desta tabela devem ser multiplicados pela fração $\hat{\sigma}^{2} / r$.

\begin{tabular}{|c|c|c|c|c|}
\hline \multirow{2}{*}{ - Valor de $\mathrm{P}$} & \multirow{2}{*}{ Valor de $\delta$} & \multicolumn{3}{|c|}{ Estimativas das Variâncias } \\
\hline & & $\hat{\boldsymbol{B}}_{i}$ & $\hat{\beta}_{i i}$ & $\vec{B}_{12}$ \\
\hline 1 & 0,951003 & 0,106085 & 0,407522 & 0,191317 \\
\hline 2 & 0,992380 & 0,100919 & 0,343690 & 0,183325 \\
\hline 3 & 1,034810 & 0,095923 & 0,290694 & 0,174825 \\
\hline 4 & 1,078878 & 0,091043 & 0,246031 & 0,165775 \\
\hline 5 & 1,125313 & 0,086222 & 0,207867 & 0,156119 \\
\hline 6 & 1,175088 & 0,081400 & 0,174822 & 0,145772 \\
\hline 7 & 1,229594 & 0,076503 & 0,145825 & 0,134612 \\
\hline 8 & 1,290994 & 0,071429 & 0,120000 & 0,122449 \\
\hline 9 & 1,363035 & 0,066019 & 0,096572 & 0,108962 \\
\hline 9 & 5,490185 & 0,005410 & 0,000367 & 0,000732 \\
\hline 10 & 1,453327 & 0,059977 & 0,074718 & 0,093529 \\
\hline 10 & 3,726639 & 0,011451 & 0,001728 & 0,003409 \\
\hline 11 & 1,582690 & 0,052550 & 0,053125 & 0,074561 \\
\hline 11 & 2,856880 & 0,018878 & 0,005004 & 0,009623 \\
\hline 12 & 2,000000 & 0,035714 & 0,020833 & 0,035714 \\
\hline
\end{tabular}


Analisando a Tabela 5, pode-se ver:

1) $\hat{\mathrm{V}}\left(\bar{\beta}_{i i}\right)>\hat{\mathrm{V}}\left(\hat{\beta}_{12}\right)>\hat{\mathrm{V}}\left(\bar{\beta}_{i}\right)$ para valores de $\mathrm{P}$ compreendidos no intervalo de 1 a 7 , mostrando que os termos quadráticos são estimados com menor precisão do que os demais, nesse intervalo.

2) $\hat{V}\left(\hat{\beta}_{i i}\right)<\hat{V}\left(\bar{B}_{12}\right)$ para valores de $P$ compreendidos no interva1o de 8 a 12 , mostrando que nesse intervalo os termos quadräticos são estimados com maior precisão do que a interação.

3) Quando $P \geq 9, \hat{V}\left(\bar{\beta}_{i i}\right)<\hat{V}\left(\bar{B}_{i}\right)$ conforme os valores de $\delta$ esco1hidos, mostrando que os termos quadräticos podem ser estimados com maior precisão do que os termos lineares.

4) Quando $P=11$ ou 12, os termos quadräticos são estimados com maior precisão do que os termos lineares, exceto quando $\delta=$ $=1,582690$ e, neste caso, pode-se considerar praticamente igual à precisão destes termos.

5) Para $P=9, \delta=5,490185$ e $r=1$, as estimativas são mais precisas.

As consideraçoes efetuadas a respeito das estimativas das variāncias das estimativas dos coeficientes do polinômio do segundo grau na Tabela 5 não levaram em conta a 
técnica preconizada por PIMENTEL GOMES e CAMPOS (1972), os quais mostraram que ao se fazer comparações de eficiências deve-se igualar os comprimentos dos intervalos. Assim, para utilizar essa técnica no presente estudo, é necessārio multi plicar as coordenadas dos pontos do delineamento por $1 / \delta$, exceto quando $\delta$ é menor do que 1 . Consequentemente, cada estimativa da variância da estimativa do coeficiente do polinô mio do segundo grau (Tabela 5) ficará multiplicada por $\delta^{2}, \delta^{4}$ e $\delta^{4}$ correspondentes a $\hat{V}\left(\hat{\beta}_{i}\right), \hat{V}\left(\hat{\beta}_{i i}\right)$ e $\hat{V}\left(\hat{\beta}_{12}\right)$, respectivamente (Tabela 6$)$.

Assim, obtem-se as seguintes fórmulas das estimativas dos coeficientes polinomiais, para $P>2$ :

$$
\hat{\mathrm{V}}\left(\bar{\beta}_{i}\right)=\frac{1}{r\left(\frac{4}{\delta^{2}}+6\right)} \cdot \bar{\sigma}^{2} \quad ; \hat{\mathrm{V}}\left(\hat{\beta}_{i i}\right)=\frac{1}{3 r} \hat{\sigma}^{2} ; \hat{\mathrm{V}}\left(\hat{\beta}_{12}\right)=\frac{1}{r\left(\frac{4}{\delta^{4}}+\frac{3}{2}\right)} \bar{\sigma}^{2} .
$$

Nota-se que quando aumenta-se o valor de $\delta$, as estimativas das variâncias $\widehat{B}_{i}$ e $\bar{\beta}_{12}$ aumentam, enquanto que a $\hat{V}\left(\bar{\beta}_{i i}\right)$ permanece constante.

Pode-se notar pela Tabela 5 que as estimativas das variâncias das estimativas dos coeficientes do polinómio do segundo grau tornam-se menores à medida que o valor de aumenta, levando a crer, à primeira vista, que a precisão das estima tivas è melhor para um alto valor de . Entretanto, utilizando a técnica de PIMENTEL GOMES e CAMPOS (1972), pode-se verificar 
na Tabela 6 que a precisão das estimativas dos coeficientes de $\beta_{i}$ e $\beta_{12}$ é melhor quando $P=2$, enquanto que no caso da precisão da estimativa do coeficiente de $\beta_{i}$ é melhor quando $P$ é maior do que 2 .

Para que se possa comparar a eficiēncia deste delineamento com os esquemas fatoriais de $3^{2}, 5^{2}$ e $7^{2}$, onde os niveis de dois fatores estão codificados em $-1,0,+1,-1,-1 / 2$, $0,+1 / 2,+1$ e $-1,-2 / 3,-1 / 3,0,+1 / 3,+2 / 3$ e +1 , respcctivamen te, é necessärio ajustar aos dados provenientes destes esquemas fatoriais o seguinte modelo matemático:

$$
\begin{aligned}
Y_{u}-\bar{Y}=\beta_{1} X_{1 u} & +\beta_{2} X_{2 u}+\beta_{11}\left(X_{1 u}^{2}-\bar{X}_{1}^{2}\right)+\beta_{22}\left(X_{2 u}^{2}-\bar{X}_{2}^{2}\right)+ \\
& +\beta_{12} X_{1 u} X_{2 u}+e_{u} .
\end{aligned}
$$

Conforme foi estudado por BATISTA (1976), no ca so de duas variáveis, o esquema fatorial $3^{2}$ é igual ao composto central ortogonal com duas variäveis. Assim sendo, quando se com para este Delineamento em Ângulos com o fatorial $3^{2}$ automaticamente compara-se este delineamento com o composto central ortogonal de duas variảveis.

Portanto, obtem-se facilmente as matrizes X'X. Assim, para o fatorial $3^{2}$, vem: 
Tabela 6. Comparação entre as estimativas das variâncias das estimativas dos coeficientes do polinomio do segundo grau, considerando o mesmo intervalo de comprimento, no Delineamento em Ângulos. Os valores desta tabe1a devem ser multiplicados pela fração $\hat{\sigma}^{2} / r$.

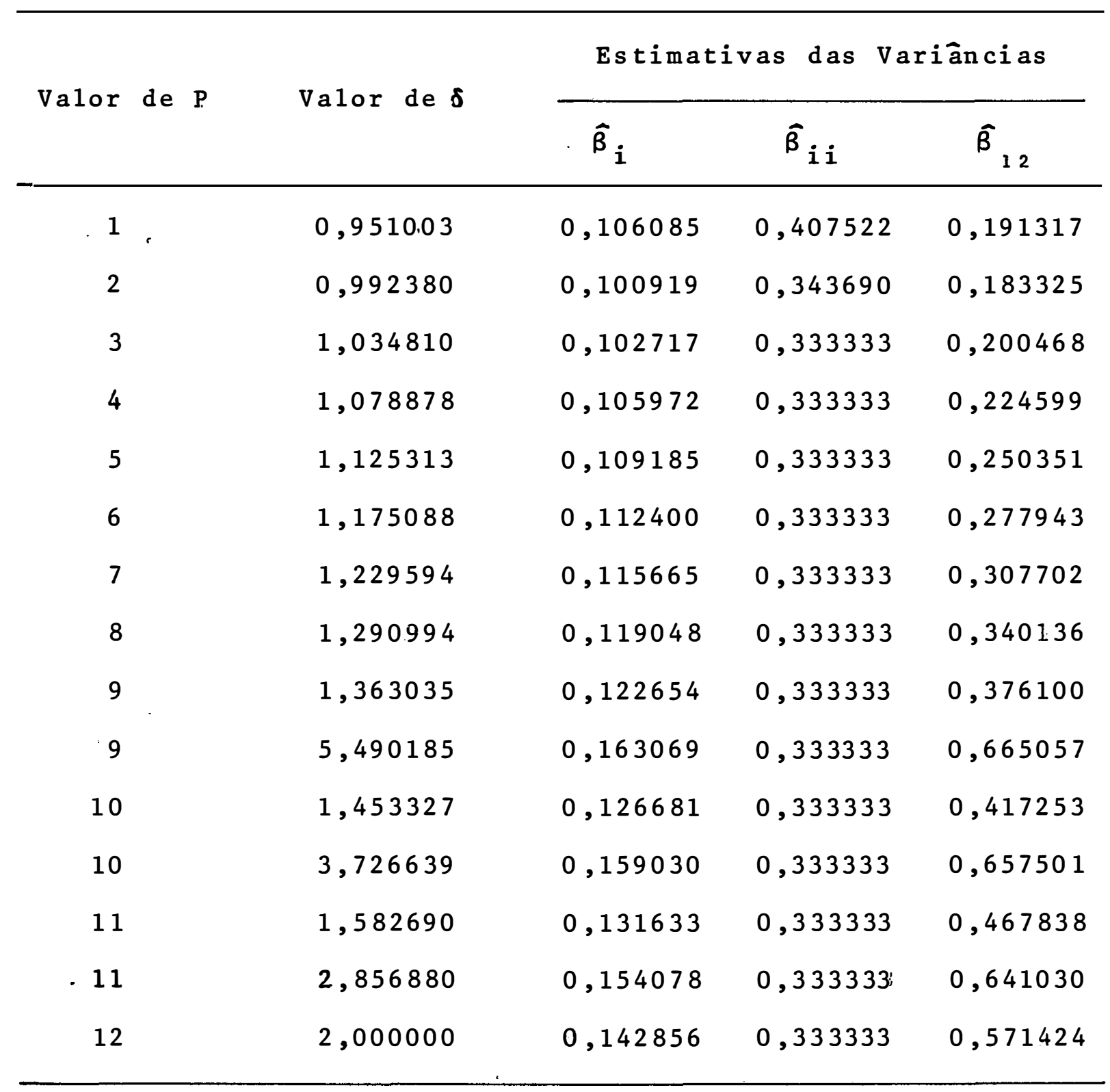


Matriz $X$ do Fatorial $3^{2}$

$\mathrm{X}=\left[\begin{array}{rrrrr}1 & 1 & 1 / 3 & 1 / 3 & 1 \\ 1 & 0 & 1 / 3 & -2 / 3 & 0 \\ 1 & -1 & 1 / 3 & 1 / 3 & -1 \\ 0 & 1 & -2 / 3 & 1 / 3 & 0 \\ 0 & 0 & -2 / 3 & -2 / 3 & 0 \\ 0 & -1 & -2 / 3 & 1 / 3 & 0 \\ -1 & 1 & 1 / 3 & 1 / 3 & -1 \\ -1 & 0 & 1 / 3 & -2 / 3 & 0 \\ -1 & -1 & 1 / 3 & 1 / 3 & 1\end{array}\right]$

Matriz $X^{\prime} X$ do fatorial $3^{2}$

$X^{\prime} X=\left[\begin{array}{lllll}6 & 0 & 0 & 0 & 0 \\ 0 & 6 & 0 & 0 & 0 \\ 0 & 0 & 2 & 0 & 0 \\ 0 & 0 & 0 & 2 & 0 \\ 0 & 0 & 0 & 0 & 4\end{array}\right]$

Para o esquema fatorial $5^{2}$ vem : 
Matriz $X$ do fatorial $5^{2}$

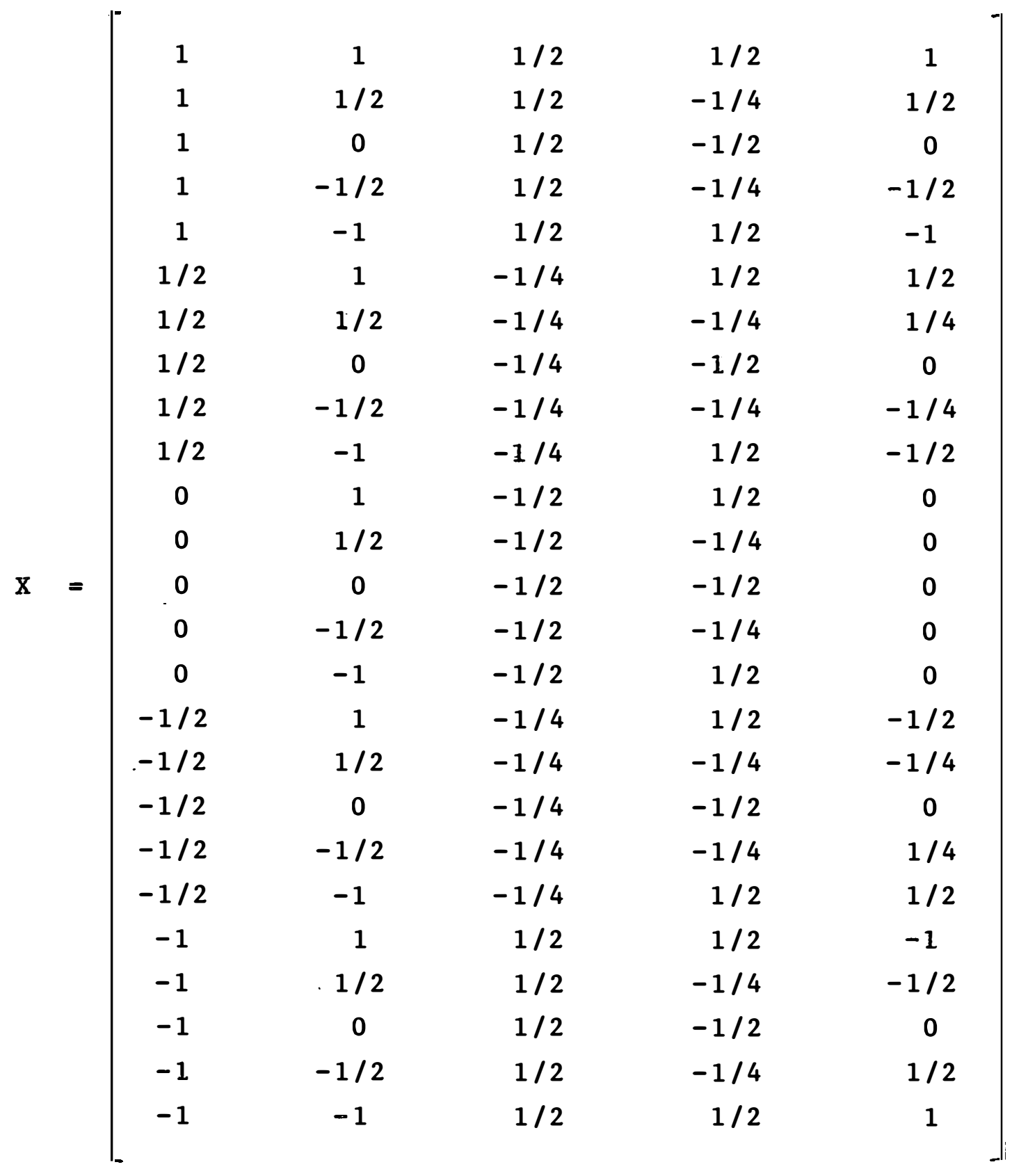


Matriz $X^{\prime} X$ do fatorial $5^{2}$

$\mathrm{X}^{\prime} \mathrm{X}=\left[\begin{array}{ccccc}25 / 2 & 0 & 0 & 0 & 0 \\ 0 & 25 / 2 & 0 & 0 & 0 \\ 0 & 0 & 35 / 8 & 0 & 0 \\ 0 & 0 & 0 & 35 / 8 & 0 \\ 0 & 0 & 0 & 0 & 25 / 4\end{array}\right]$

No esquema fatorial $7^{2}$ tem-se: 
Matriz $X$ do fatorial $7^{2}$

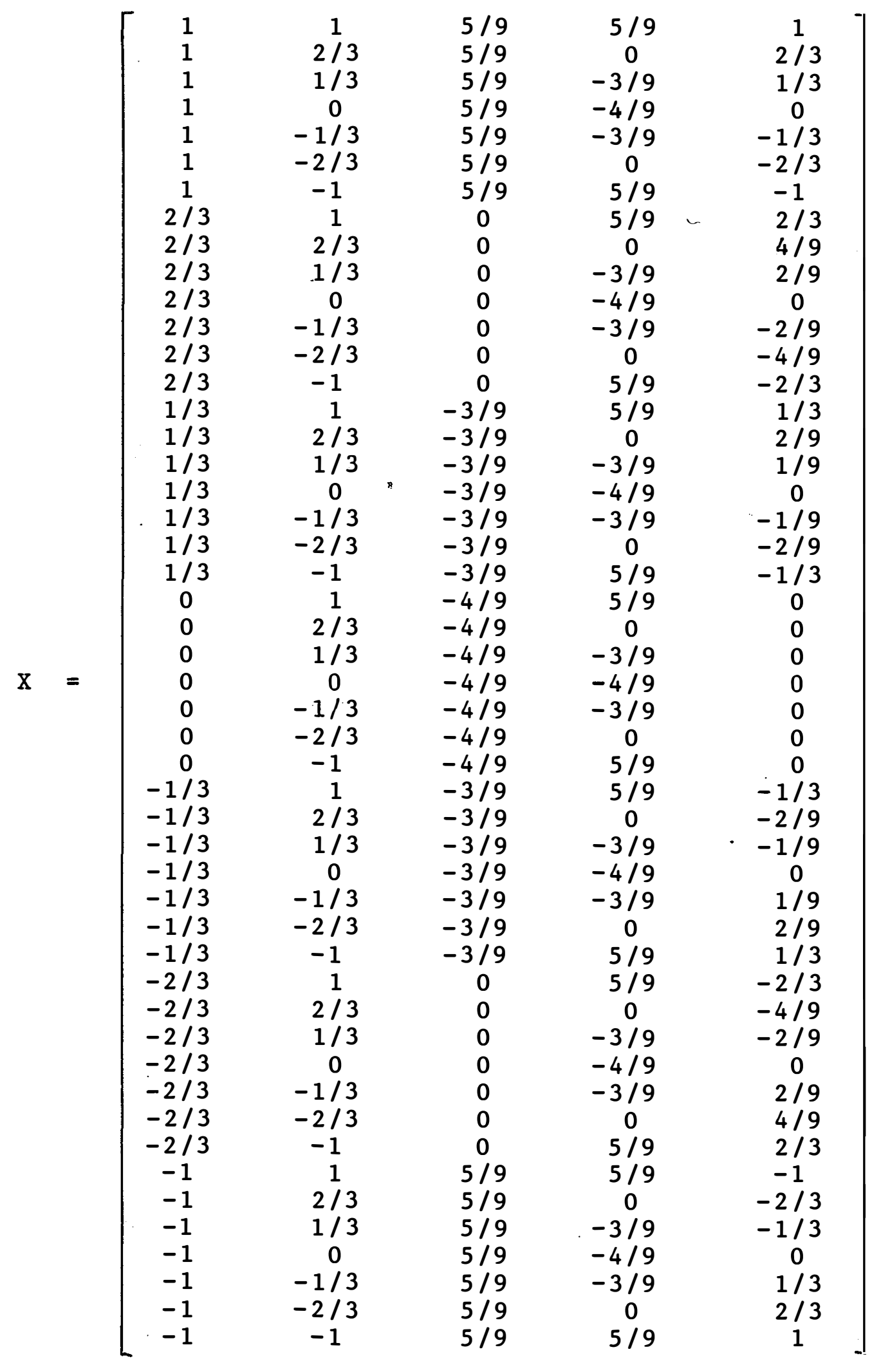


Matriz $X^{\prime} X$ do fatorial $7^{2}$

$X^{\prime} X=\left[\begin{array}{ccccc}196 / 9 & 0 & 0 & 0 & 0 \\ 0 & 196 / 9 & 0 & 0 & 0 \\ 0 & 0 & 588 / 81 & 0 & 0 \\ 0 & 0 & 0 & 588 / 81 & 0 \\ 0 & 0 & 0 & 0 & 784 / 81\end{array}\right]$

Consequentemente, as variāncias das estimativas dos coeficientes do polinômio do segundo grau, nos très esquemas fatoriais, são dadas pelas seguintes expressões.

Tabela 7 - Variāncias das estimativas dos coeficientes polincmiais nos esque mas Fatoriais $3^{2}, 5^{2}$ e $7^{2}$.

\begin{tabular}{cccc} 
Fatorial & $V\left(\hat{\beta}_{i}\right)$ & $V\left(\hat{\beta}_{i i}\right)$ & $V\left(\hat{\beta}_{12}\right)$ \\
$3^{2}$ & $\frac{1}{6 r} \cdot \sigma^{2}$ & $\frac{1}{2 r} \cdot \sigma^{2}$ & $\frac{1}{4 r} \cdot \sigma^{2}$ \\
$5^{2}$ & $\frac{2}{25 r} \cdot \sigma^{2}$ & $\frac{8}{35 r} \cdot \sigma^{2}$ & $\frac{4}{25 r} \cdot \sigma^{2}$ \\
$7^{2}$ & $\frac{81}{196 r} \cdot \sigma^{2}$ & $\frac{88 r}{85} \cdot \sigma^{2}$ & $\frac{784}{78}$ \\
\hline
\end{tabular}


Comparando-se estas variâncias com as variâncias das estimativas dos coeficientes do polinômio do segundo grau ajustadas para o mesmo intervalo de comprimento (Tabela 6), verifica-se:

1) O Delineamento em Ângulos é mais preciso do que o Fato rial $3^{2}$ e menos preciso do que os Fatoriais $5^{2}$ e $7^{2}$ quando se considera a estimativa de $\beta_{1}$.

2) 0 Delineamento em Ângulos é mais preciso do que o Fato rial $3^{2}$ e menos preciso do que os Fatoriais $5^{2}$ e $7^{2}$ na estimativa de $\beta_{i i}$.

3) Na estimativa de $\beta_{12}$, o Delineamento em Ângulos somente é mais preciso do que o Fatorial $3^{2}$ quando se utiliza até um máximo de quatro pontos centrais. Comparado com os Fatoriais $5^{2}$ e $7^{2}$, o Delineamento em Ângulos é menos preciso na estimação do coeficiente $\beta_{12}$.

Nas comparações anteriores não se levou em consideração que na experimentação a ser efetuada se deve ter o mesmo custo em termos da ärea total do experimento. Assim, para tornar a comparação equitativa, igualou-se o número de parcelas em todos os delineamentos considerados. 
Para tanto, tomou-se como referência o fatorial $7 \times 7$ com 49 parcelas, por ser este o nümero máximo de parcelas encolvido nas comparações aqui efetivadas. No entanto poderia se ter escolhido como base qualquer outro dos delineamentos em estudo.

Assim; tem-se que repetir os esquemas fatoriais tantas vezes quantos forem os resultados das razões $49 / 9$; $49 / 25$ e 49/49, respectivamente, aos esquemas fatoriais d $33^{2}, 5^{2}$ e $7^{2}$ (Tabela 8). Quanto ao presente delineamento, as repetições serão dadas pelas razões $49 /(16+P)$ (Tabela 9).

Tabela 8 - Variāncias das estimativas dos coeficientes polinomiais nos esquemas fatoriais de $3^{2}, 5^{2}$ e $7^{2}$, conside rando a mesma ärea total.

\begin{tabular}{|c|c|c|c|}
\hline FATORIAL & $v\left(\bar{\beta}_{i}\right)$ & $v\left(\hat{\beta}_{i}\right)$ & $\mathbf{V}(\hat{B})$ \\
\hline $3^{2}$ & $0,030612 \sigma^{2}$ & $0,091837 \sigma^{2}$ & $0,045918 \sigma^{2}$ \\
\hline $5^{2}$ & $0,040816 \sigma^{2}$ & $0,116618 \sigma^{2}$ & $0,081633 \sigma^{2}$ \\
\hline $7^{2}$ & $0,045918 \sigma^{2}$ & $0,137755 \sigma^{2}$ & $0,103316 \sigma^{2}$ \\
\hline
\end{tabular}


Tabela 9 - Variāncias das estimativas dos coeficientes polinomiais no Delineamento em Ângulos, considerando-se o mesmo intervalo de comprimento e a mesma ärea total utilizada. Os valores desta tabela devem ser multiplicados por $\sigma^{2}$.

\begin{tabular}{cccccc}
\hline & & & \multicolumn{3}{c}{ VARIANCIAS } \\
\cline { 3 - 5 } 1 & VALOR DE & $\delta$ PALOR & $\bar{B}_{i}$ & $\bar{\beta}_{12}$ \\
\hline 2 & 0,951003 & 0,036805 & 0,141385 & 0,066375 \\
3 & 0,992380 & 0,037072 & 0,126253 & 0,067344 \\
4 & 1,034810 & 0,039829 & 0,129252 & 0,077732 \\
5 & 1,078878 & 0,043254 & 0,136054 & 0,091673 \\
6 & 1,125313 & 0,046794 & 0,142857 & 0,107293 \\
7 & 1,175088 & 0,050465 & 0,149660 & 0,124791 \\
8 & 1,229594 & 0,054292 & 0,156462 & 0,144432 \\
9 & 1,290994 & 0,058309 & 0,163265 & 0,166597 \\
9 & 1,363035 & 0,062579 & 0,170068 & 0,191888 \\
10 & 5,490185 & 0,083198 & 0,170068 & 0,339315 \\
10 & 1,453327 & 0,067218 & 0,176871 & 0,221400 \\
11 & 3,726639 & 0,084383 & 0,176871 & 0,348878 \\
11 & 1,582690 & 0,072532 & 0,183673 & 0,257788 \\
12 & 2,856880 & 0,084900 & 0,183673 & 0,353221 \\
& 2,000000 & 0,081632 & 0,190476 & 0,326528 \\
\hline & & & & & \\
\hline
\end{tabular}


Estudando a Tabela 9 , verifica-se que quanto aos coeficientes $\hat{\beta}_{i}$ e $\hat{\beta}_{12}$, a precisão máxima é atingida com um ponto central. Quanto ao coeficiente $\hat{\beta}_{i}$, a precisão máxima è obtida quando são usados dois pontos centrais. No entanto, com um ponto central, a precisão de $\bar{\beta}_{i i}$ está bem próxima daquela ob tida com dois pontos centrais. Assim, considerando-se os parâme $\operatorname{tros} \bar{\beta}_{i}, \bar{\beta}_{i i}$ e $\bar{\beta}_{12}$, verifica-se que o delineamento é mais preciso quando se usa somente um ponto central.

Comparando-se o Delineamento em Ângulos com um ponto central (Tabela 9) com os esquemas Fatoriais de $3^{2}, 5^{2}$ e $7^{2}($ Tabela 8$)$, notou-se que:

1. Quanto ao coeficiente $\hat{B}_{i}$, o delineamento em questão é tão preciso quanto o esquema fatorial $3^{2}$ e mais preciso do que os fatoriais $5^{2}$ e $7^{2}$.

2. Quanto ao coeficiente $\hat{B}_{i}$, o delineamento em àngulos com um ponto central é menos preciso do que os fatoriais de $3^{2}, 5^{2}$ e $7^{2}$.

3. Quanto ao coeficiente $\hat{\beta}_{12}$, o delineamento em ângulos com um ponto central é menos preciso do que o fatorial $3^{2}$ e mais preciso do que os fatoriais de $5^{2}$ e $7^{2}$.

As estimativas dos coeficientes $B_{i}, \beta_{i i}$ e $\beta_{12}$ são determinadas matricialmente por:

$$
\hat{B}=\left(X^{\prime} X\right)^{-1} X^{\prime} Y \text {. }
$$


Como $\left(X^{\prime} X\right)^{-1}$ é diagonal, facilmente se determi nam as fórmulas que permitem calcular as estimativas dos coeficientes do polinomio do segundo grau, no presente delineamento ortogonal. Assim, temos:

$$
\begin{aligned}
& \hat{\beta}_{i}=\frac{\sum_{u} x_{i u} y_{u}}{r\left(4+6 \delta^{2}\right)} \quad \hat{\beta}_{i i}=\frac{\sum_{u} x_{i u}^{2} y_{u}-\frac{\left(4+6 \delta \delta^{2}\right)_{u} y_{u}}{16+P}}{3 r \delta^{4}} \\
& \hat{\beta}_{12}=\frac{\sum_{u} x_{1 u} x_{2 u^{y}}{ }^{\dddot{y}}}{r\left(4+3 / 2 \delta^{4}\right)}
\end{aligned}
$$

onde $x_{i u}, x_{i u}^{2}$ e $x_{1 u} x_{2 u}$ são os valores encontrados nas colu nas da matriz $x$.

Comparando o modelo matemático:

$$
\hat{Y}_{u}=\hat{\beta}_{0}+\hat{\beta}_{1} X_{1 u}+\hat{\beta}_{2} X_{2 u}+\hat{\beta}_{11} X_{1 u}^{2}+\hat{\beta}_{22} X_{2 u}^{2}+\hat{\beta}_{12} X_{1 u} X_{2 u} \quad \text { (e.7) }
$$

com o modelo matemáticoadotado: 


$$
\begin{gathered}
\hat{Y}_{u}-\bar{Y}=\hat{\beta}_{1} X_{1 u}+\hat{\beta}_{2} X_{2 u}+\hat{\beta}_{11}\left(x_{1 u}^{2}-\bar{x}_{1}^{2}\right)+\hat{\beta}_{22}\left(x_{2 u}^{2}-\bar{x}_{2}^{2}+\right. \\
+\hat{\beta}_{12} X_{1 u} X_{2 u} \\
\quad(e .8)
\end{gathered}
$$

Pode-se facilmente obter $\hat{\beta}_{0}$, pois da expressão 8 tem-se:

$$
\begin{aligned}
\hat{\mathrm{Y}}_{u}= & \bar{Y}+\hat{\beta}_{1} X_{1 u}+\hat{\beta}_{2} X_{2 u}+\hat{\beta}_{11}\left(x_{1 u}^{2}-\bar{x}_{1}^{2}\right)+\hat{\beta}_{22}\left(X_{2 u}^{2}-\bar{x}_{2}^{2}\right)+ \\
& +\hat{\beta}_{12} X_{1 u} X_{2 u}
\end{aligned}
$$

$$
\begin{aligned}
& \text { Assim, igualando a expressão } 7 \text { à } 9 \text {, vem : } \\
& \hat{\beta}_{0}=\bar{Y}-\hat{\beta}_{11} \bar{x}_{1}^{2}-\hat{\beta}_{22} \bar{X}_{2}^{2}
\end{aligned}
$$$$
\text { Mas como } \frac{\bar{x}_{1}^{2}}{1}=\overline{\mathrm{x}}_{2}^{2}=\frac{4+6 \delta^{2}}{16+\mathrm{P}}, \quad \text { logo: }
$$$$
\hat{\beta}_{0}=\bar{Y}-\frac{4+6 \delta^{2}}{16+P}\left(\hat{\beta}_{11}+\hat{B}_{22}\right)
$$ 
A soma de quadrados devida à regressão é dada por $\hat{B}^{\prime} X^{\prime} Y$. Assim, as somas dos quadrados referentes aos coeficientes são dadas por:

$$
\begin{aligned}
& \operatorname{SQ}\left(\hat{\beta}_{i}\right)=\hat{\beta}_{i} \sum_{u} x_{i u} y_{u} \\
& \operatorname{sQ}\left(\hat{\beta}_{i i}\right)=\hat{\beta}_{i i}\left[\sum_{u} x_{i u}^{2} y_{u}-\frac{\left(4+6 \delta^{2}\right) \sum_{u} y_{u}}{16+P}\right] \\
& \operatorname{sQ}\left(\hat{\beta}_{12}\right)=\hat{\beta}_{12} \sum_{u} x_{u} x_{u} y_{u}
\end{aligned}
$$

Quando se desejar repetir os tratamentos é recomendado por BATISTA (1978) utilizar somente um ponto central, pois torna-se mais simples o quadro de análise de variância. Se assim for feito e se, por exemplo, for adotado o delineamento em blocos ao acaso, obter-se-a a decomposição para a análise de variância com um ponto central, conforme consta da Tabe 1 a 10 .

Quando se desejar repetir somente o ponto central, recomenda-se, devido às tēcnicas experimentais, utilizar 11 ou 12 pontos centrais, pois assim, haverä no minimo 10 graus de 1 iberdade para o residuo.Nes se caso, a decomposição resulta como na Tabela 11. A Tabela 12 mostra a decomposição para anālise de variāncia quando se desejar repetir pontos centrais e tratamentos. 


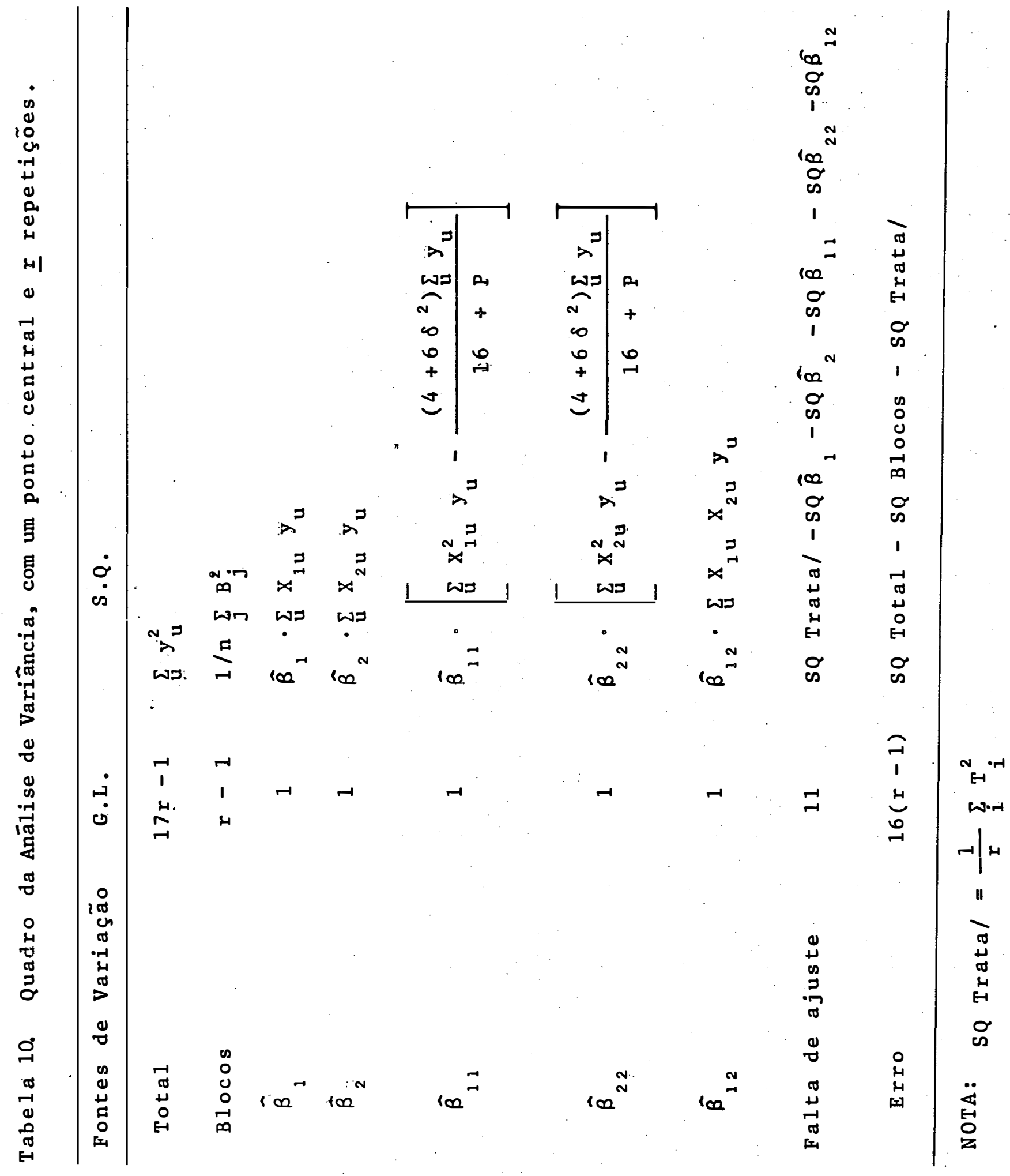




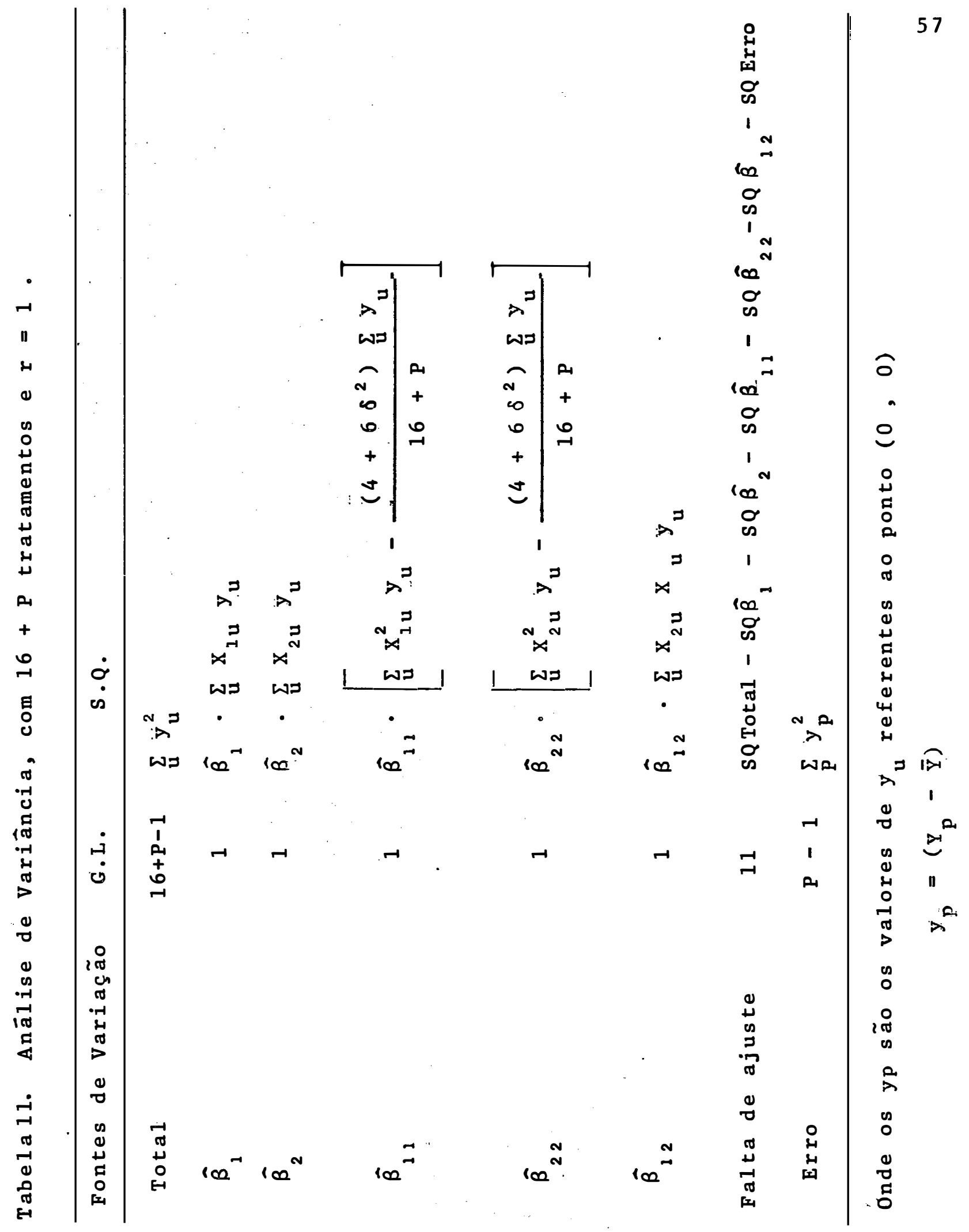




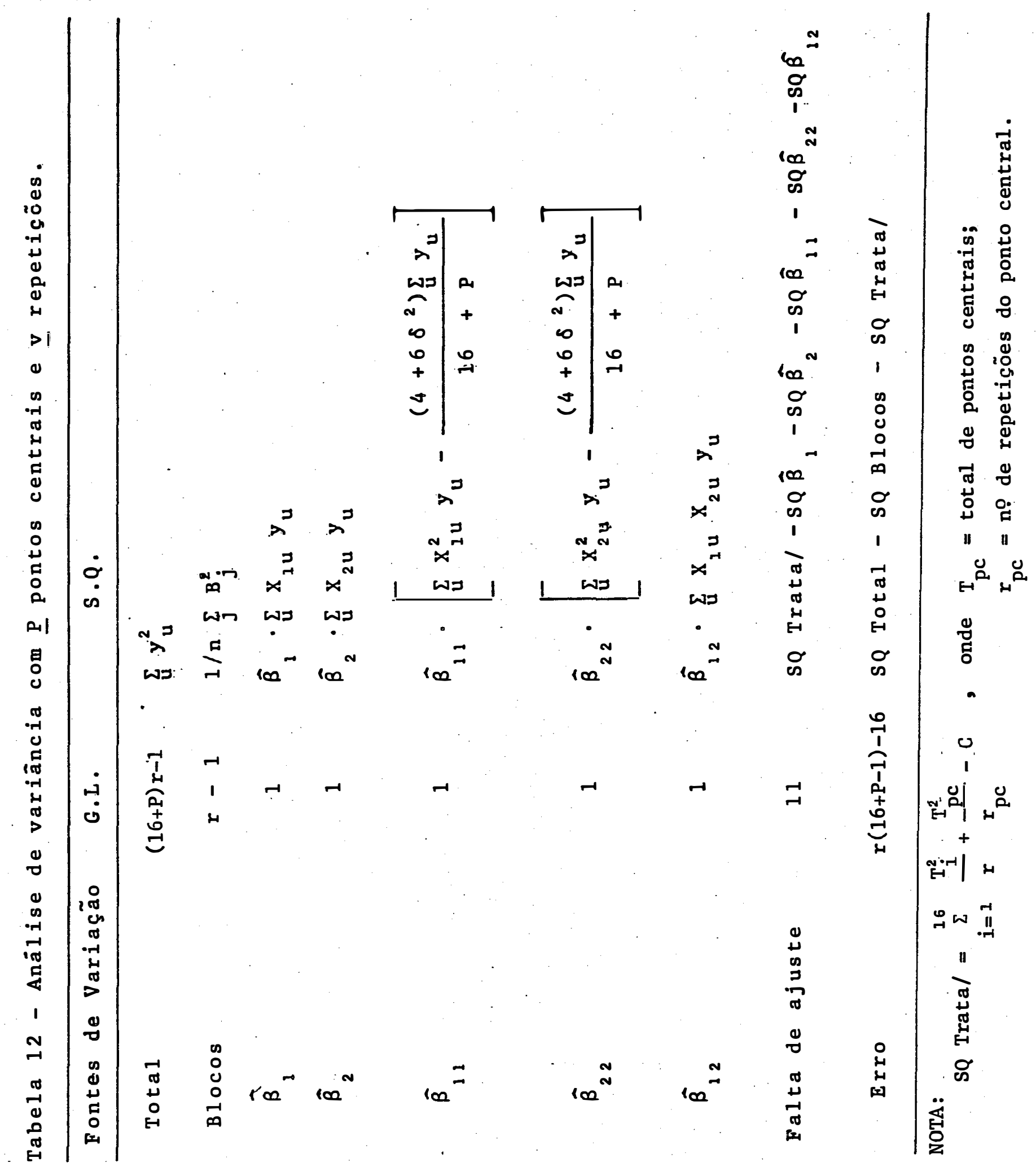


Apōs ajustada a equação do polinômio do segundo grau aos dados obtidos, pode-se aplicar a técnica de congruência de matrizes para verificar se a função tem máximo ou mínimo ou ponto de sela (PIMENTEL GOMES, 1969). 


\section{EXEḾPLO DE APLICAÇÃO}

Para exemplificar a aplicação da metodologia da superfície de resposta polinomial a dados provenientes de experimentos com o delineamento em ângulos, foi simulado um ensaio com dois fatores em blocos ao acaso.

Os dados foram obtidos a partir da equação fun damental, de segundo grau (Tabela 6). Aos valores esperados, a partir dessa equação, foram adicionados erros provenientes de distribuição normal. 
Tabela 13. Dados de um Delineamento em Ângulos, em blocos ao acaso, com um ponto central e quatro repetiçöes.

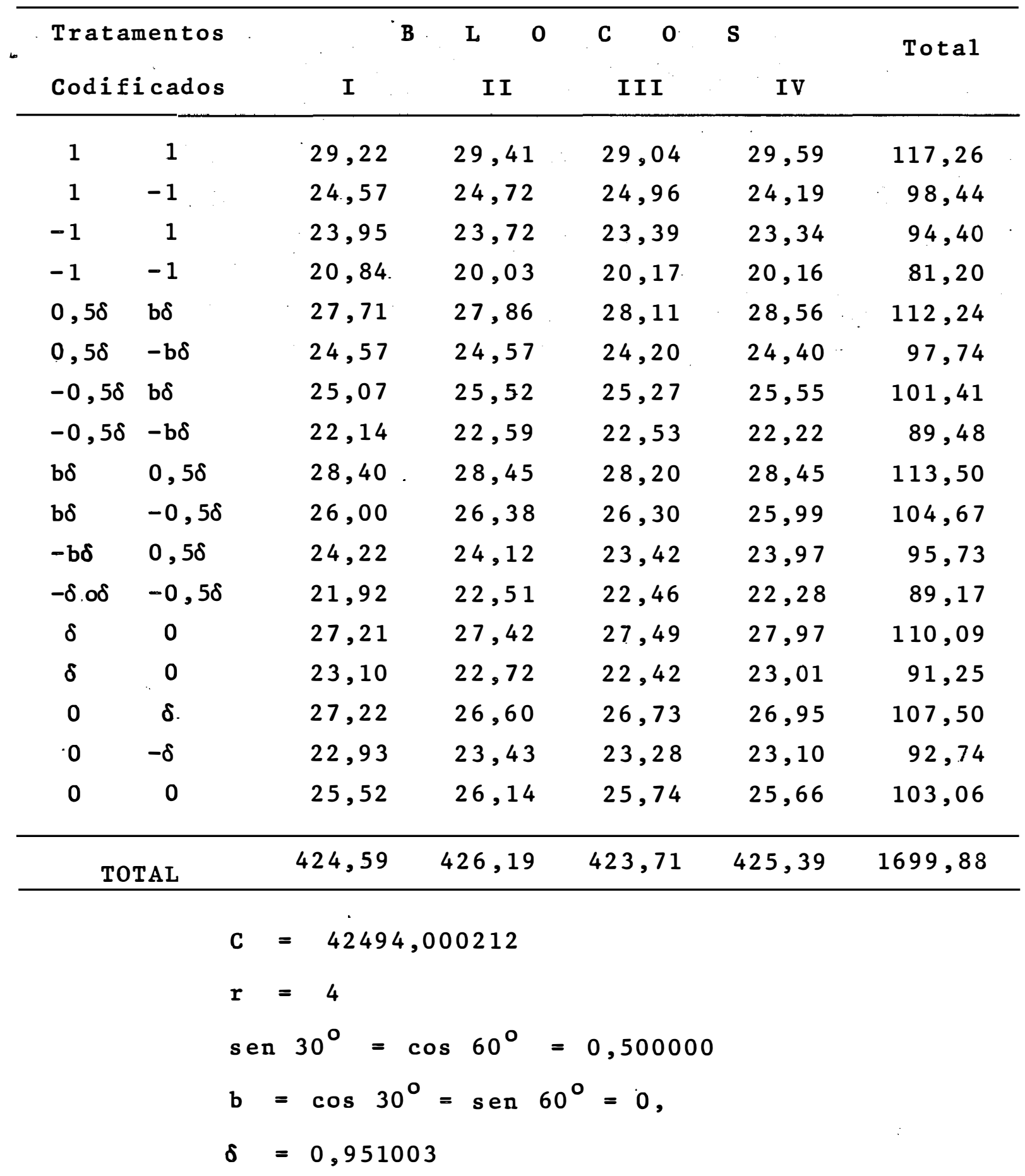


Figura 3. Delineamento em Āngulos, $\operatorname{com} P=1$.

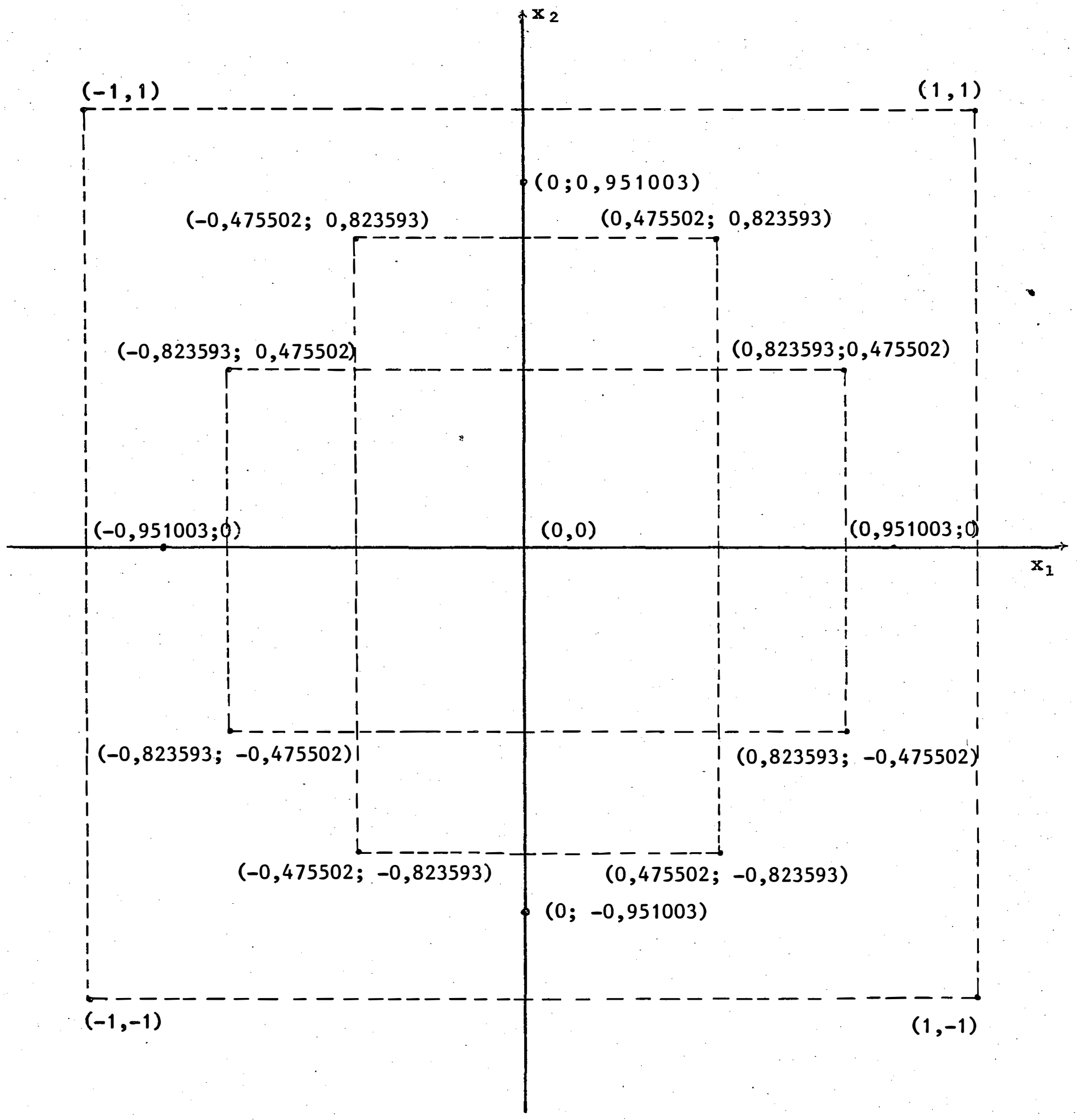

Nota: $0,951003=\delta$ $(0,475502 ; 0,823593)=\left(\delta \cos 60^{\circ} ; \delta \operatorname{sen} 60^{\circ}\right)$

- demais pontos como na figura 2. 
5.1. Determinação dos coeficientes do polinômio do segundograu

$$
\begin{aligned}
& 5.1 .1 \cdot \widehat{B}_{1} \\
& \widehat{B}_{1}=\frac{\sum_{u} x_{1 u} \dot{y}_{u}}{r\left(4+6 \delta^{2}\right)}=\frac{94,495138}{37,705761}=2,506119
\end{aligned}
$$

$$
\begin{aligned}
& 5.1 .2 \cdot \hat{B} \\
& \hat{B}_{2}=\frac{\sum_{u} x_{2 u} y_{u}}{r\left(4+6 \delta^{2}\right)}=\frac{75,142319}{37,705761}=1,992860
\end{aligned}
$$

$5.1 \cdot 3 \cdot \hat{\beta}_{11}$

$$
\widehat{B}_{I 1}=\frac{\sum_{u} x_{I u}^{2} y_{u}-\frac{\left(4+6 \delta^{2}\right) \sum_{u} y_{u}}{16+P}}{3 r \delta^{4}}
$$

$$
\hat{\boldsymbol{B}}_{11}=\frac{937,434742-942,577484}{9,815418}=\frac{-5,142742}{9,815418}=-0,523945
$$




$$
\begin{aligned}
& 5.1 .4 \cdot \hat{\beta}_{22} \\
& \widehat{B}_{22}=\frac{\sum_{u} x_{2 u}^{2} y_{u}-\frac{\left(4+6 \delta^{2}\right) \sum_{u} y_{u}}{16+P}}{3 \delta^{4}} \\
& \widehat{B}_{22}=\frac{935,445085-942,577484}{9 ., 815418}=-\frac{-7,132399}{9,815418}=-0,726653
\end{aligned}
$$

$$
\begin{aligned}
& 5.1 .5 . \hat{\beta}_{12} \\
& \hat{\beta}_{12}=\frac{\sum_{u} x_{1 u} x_{2 u} y_{u}}{r\left(4+1,5 \delta^{4}\right)}=\frac{7,515438}{20,907709}=0,359458
\end{aligned}
$$

$$
\begin{aligned}
& 5.1 .6 . \hat{B}_{0} \\
& \hat{B}_{0}=\bar{Y}_{u}-\frac{4+6 \delta^{2}}{16+P}\left(\hat{\beta}_{11}+\hat{B}_{22}\right) \\
& \hat{B}_{0}=24,998235-0,554496(-0,523945-0,726653) \\
& \hat{\beta}_{0}=24,998235+(0,554496 \times 1,250598)=25,691687
\end{aligned}
$$


5.2. Determinação das somas de quadrados

$5.2 .1 . S Q$ Total

SQ Total $=\sum_{\mathrm{u}} \mathrm{y}_{\mathrm{u}}^{2}=i_{\mathrm{j}}^{\sum_{\mathrm{i} j}} \mathrm{Y}_{\mathrm{j}}^{2}-\mathrm{C}$

SQ Total $=42895,121400-42494,000212$

SQTotal = 401,121188

$5.2 .2 . \mathrm{SQB} 10 \cos$

$S Q B 1 \dot{c o s}=\frac{1}{n} \sum_{j} B_{j}^{2}-C=42494,200024-42494,000212=0,199812$

5.2.3. SQ Tratamentos

SQ Tratamentos $=\frac{1}{r} \sum_{i} T_{i}^{2}-C$

SQ Tratamentos $=42891,249350-42494,000212=397,249138$ 
$5.2 \cdot 4 \cdot S Q \hat{B}_{1}$

$\operatorname{SQ} \hat{B}_{1}=\hat{B}_{1} \cdot \sum_{\mathbf{u}} \mathrm{X}_{1 \mathrm{u}} \mathrm{y}_{\mathbf{u}}=2,506119 \times 94,495138=236,816061 \cdots$

$5.2 .5 . \quad S Q \hat{B}_{2}$

$\operatorname{SQ} \hat{\beta}_{2}=\hat{\beta}_{2} \cdot \sum_{u} X_{2 u} y_{u}=1,992860 \times 75,142319=149,748122$

$5.2 .6 . \quad S Q \hat{B}_{11}$

$\operatorname{SQ} \hat{\beta}_{11}=\hat{\beta}_{11} \cdot\left[\sum_{\mathrm{u}} \mathrm{x}_{1 \mathrm{u}}^{2} \mathrm{y}_{\mathrm{u}}-\frac{\left(4+6 \delta^{2}\right) \sum_{\mathrm{u}} \mathrm{y}_{\mathrm{u}}}{16+\mathrm{P}}\right]$

$\mathrm{SQB}_{11}=-0,523945 \cdot[937,434742-942,577484]$

$\operatorname{SQ} \hat{B}_{11}=-0,523945 \times(-5,142742)=2,694514$

$5.2 .7 \cdot S_{22}$

$\operatorname{sQ} \hat{\beta}_{22}=\hat{\beta}_{22} \cdot\left[\sum_{u} x_{2 u}^{2} y_{u}=\frac{-\left(4+6 \delta^{2}\right) \sum_{u} y_{u}}{16+P}\right]$ 


$$
\begin{aligned}
& \operatorname{SQ}_{22}=-0,726653 \cdot[935,445085-942,577484] \\
& \operatorname{SQS}_{22}=-0,726653 \times(-7,132399)=5,182779
\end{aligned}
$$

$5.2 .8 . \quad S Q \hat{\beta}_{12}$

$\operatorname{SQ} \hat{\beta}_{12}=\hat{\beta}_{12} \sum_{u} \dot{x}_{1 u} x_{2 u} y_{u}=0,359458 \cdot 7,515438=2,701484$

\subsubsection{SQ Falta de ajuste}

SQ Falta de ajuste $=S Q T r a t a m e n t o s-S Q \hat{B}_{1}-S Q \hat{\beta}_{2}-S Q \hat{\beta}_{11}-S Q \hat{B}_{22}-S_{12} \hat{\beta}_{12}$

SQ Falta de ajuste $=397,249138-236,816061-149,748122-2,694514-$

$$
-5,182779-2,701484
$$

SQFalta de ajuste $=0,106178$

5.2.10. SQ Erro

SQErro = SQ Total - SQ Blocos - SQ Tratamentos

SQ Erro = 401,121188-0,199812-397,249138

SQ Erro = 3,672238 
5.3. Quadro da Anālise de Variância

\begin{tabular}{|c|c|c|c|c|}
\hline F. V. & G.L : & $\mathbf{S} \cdot \mathbf{Q} \cdot$ & Q.M. & $\mathbf{F}$ \\
\hline Total & 67 & 401,121188 & & \\
\hline B locos & 3 & 0,199812 & 0,066604 & --- \\
\hline$\hat{B}_{1}$ & 1 & 236,816061 & 236,816061 & $3095,43^{* *}$ \\
\hline$\hat{\beta}_{2}$ & 1 & 149,748122 & 149,748122 & $1957,36 * *$ \\
\hline$\hat{\beta}_{11}$ & 1 & 2,694514 & 2,694514 & $35,22 * *$ \\
\hline$\hat{B}_{22}$ & 1 & 5,182779 & 5,182779 & $67,74 * *$ \\
\hline$\hat{\beta}_{12}$ & 1 & 2,701484 & 2,701484 & $35,31 * *$ \\
\hline Falta de ajuste & 11 & 0,106178 & 0,009653 & --- \\
\hline Erro & 48 & 3,672238 & 0,076505 & \\
\hline
\end{tabular}


5.4. Determinação das estimativas das variâncias das estimativas dos coeficientes polinomiais

$$
\begin{aligned}
& 5.4 .1 \cdot \hat{V}\left(\hat{\beta}_{i}\right) \\
& \hat{v}\left(\hat{\beta}_{i}\right)=\frac{1}{r\left(4+6 \delta^{2}\right)} \cdot \hat{\sigma}^{2} \\
& \delta=0,951003(\mathrm{Tabe1a} 1) \\
& \hat{\mathrm{V}}\left(\hat{\beta}_{i}\right)=\frac{0,076505}{37,705761}=0,002029
\end{aligned}
$$

$$
\begin{aligned}
& 5.4 .2 \cdot \hat{V}\left(\hat{\beta}_{i i}\right) \\
& \hat{v}\left(\hat{\beta}_{i i}\right)=\frac{1}{3 r \delta^{4}} \cdot \hat{\sigma}^{2}=\frac{0,076505}{3 \times 4 \times 0,951003^{4}}=0,007794
\end{aligned}
$$

$$
\begin{aligned}
& 5.4 .3 \cdot \hat{V}\left(\hat{\beta}_{12}\right) \\
& \hat{v}\left(\hat{\beta}_{12}\right)=\frac{1}{r\left(4+1,5 \delta^{4}\right)} \cdot \hat{\sigma}^{2}=\frac{0,076505}{20,907709}=0,003659
\end{aligned}
$$


5.5. Determinação dos valores de "t" para testar as hipóteses de que as estimativas dos coeficientes polinomiais não diferem de zero

5.5 .1$. Valor de "t" para testar ${ }_{0}^{(1)}: \beta_{1}=0$

$$
t=\frac{\hat{B}_{1}}{\sqrt{\hat{V}\left(\hat{B}_{1}\right)}}=\frac{2,506119}{\sqrt{0,002029}}=55,64
$$

Note que, de acordo $\operatorname{com} 5.3, F=t^{2}$, isto é, $t=\sqrt{F}=\sqrt{3095,43}=55,64$

5.5 .2 . Valor de " $t$ " para testar $\mathrm{H}_{\mathrm{O}}^{(2)}: \beta_{2}=0$

$$
t=\frac{\hat{\beta}_{2}}{\sqrt{\hat{V}\left(\hat{\beta}_{2}\right)^{\prime}}}=\frac{1,992860}{\sqrt{0,002029}}=44,24
$$

Note que, de acordo $\operatorname{com} 5.3, F=t^{2}$, isto é, $t=\sqrt{F}=\sqrt{1957,36}=44,24$ 
5.5.3. Valor de "t" para testar $\mathrm{H}_{0}^{(3)}: \beta_{11}=0$

$$
t=\frac{\hat{B}_{1 i}}{\sqrt{\hat{V}\left(\hat{B}_{11}\right)}}=\frac{-0,523945}{\sqrt{0,007794}}=-5,93
$$

Note que $t=-\sqrt{35,22}=-5,93$, de acordo com o item 5.3

5.5.4. Valor de " $t$ " para testar $\mathrm{H}_{\mathrm{O}}^{(4)}: \mathrm{B}_{22}=0$

$$
t=\frac{\widehat{\beta} 22}{\sqrt{\hat{V}\left(\beta_{22}\right)}}=\frac{-0,726653}{\sqrt{0,007794}}=-8,23
$$

Note que, de acordo $\operatorname{com} 5,3, t=-\sqrt{ } 67,74=-8,23$

5.5 .5$. Valor de "t" para testar $\mathrm{H}_{0}^{(5)}: \beta_{12}=0$

$$
t=\frac{\hat{\beta}_{12}}{\sqrt{\hat{V}\left(\hat{\beta}_{12}\right)}}=\frac{0,359458}{\sqrt{0,003659}}=5,94
$$

Note que $t=\sqrt{35,31}=5,94$, de acordo com 5.3 . 
5.6. Determinação da equação polinomia1

$$
\begin{aligned}
\hat{\mathrm{Y}}_{\mathrm{u}}= & 25,691687+2,506119 \mathrm{x}_{1 \mathrm{u}}+1,992860 \mathrm{x}_{2 \mathrm{u}}- \\
& -0,523945 \mathrm{x}_{1 \mathrm{u}}^{2}-0,726653 \mathrm{x}_{2 \mathrm{u}}^{2}+0,359458 \mathrm{x}_{1 \mathrm{u}} \mathrm{X}_{2 \mathrm{u}}
\end{aligned}
$$

5.7. Estudo do máximo, mínimo ou ponto de sela através de congruência de matrizes

De acordo com o método descrito em 3.7 , tem-se:

$$
\begin{aligned}
& \mathrm{B}=\left[\begin{array}{rc}
-0,523945 & 0,359458 \\
0,359458 & -0,726653
\end{array}\right] \\
& \mathrm{L}_{\hat{2}}=0,523945 \mathrm{~L}_{2}+0,359458 \mathrm{~L}_{1} \\
& \mathrm{C}_{2}=\left[\begin{array}{cc}
0,523945 & 0,359458 \\
0,523945 \mathrm{C} 2 & +0,359458 \mathrm{C} 1 \\
\mathrm{~B}= & -0,251516 \\
-0,523945 & 0 \\
0 & -0,131781
\end{array}\right]
\end{aligned}
$$

Como a matriz B e definida negativa então a fun ção atinge ponto de máximo. 
5.8. Determinação do máximo da funçãa

$$
\begin{aligned}
\vec{Y}= & 25,691687+2,506119 x_{1 u}+1,992860 x_{2 u}- \\
& -0,523945 x_{1 u}^{2}-0,726653 x_{2 u}^{2}+0,359458 x_{1 u} x_{2 u}
\end{aligned}
$$

$\frac{\mathrm{d} \hat{\mathrm{Y}}_{\mathrm{u}}}{\mathrm{d} \mathrm{X}_{1 \mathrm{u}}}=2,506119-1,047890 \mathrm{X}_{1 \mathrm{u}}+0,359458 \mathrm{X}_{2 \mathrm{u}}$

$\frac{\mathrm{d} \hat{\mathrm{Y}}_{\mathrm{u}}}{\mathrm{d} \mathrm{X}_{2 \mathrm{u}}}=1,992860-1,453306 \mathrm{x}_{2 \mathrm{u}}+0,359458 \mathrm{x}_{1 \mathrm{u}}$

Fazendo $\frac{d \hat{Y}_{u}}{d X_{1 u}}=\frac{d \hat{Y}_{u}}{d X_{2 u}}=0$, vem

$$
\left\{\begin{array}{c}
1,047890 x_{1 u}^{0}-0,359458 x_{2 u}^{0}=2,506119 \\
-0,359458 x_{1 u}^{0}+1,453306 x_{2 u}^{0}=1,992860
\end{array}\right.
$$

Resolvendo o sistema, vem:

$$
\left[\begin{array}{rr}
1,047890 & -0,359458 \\
-0,359458 & 1,453306
\end{array}\right]=\left[\begin{array}{c}
x_{1 u}^{0} \\
x_{2 u}^{0}
\end{array}\right]=\left[\begin{array}{c}
2,506119 \\
1,992860
\end{array}\right]
$$


Sendo o determinante:

$$
\begin{aligned}
& \Delta=1,393695 \text {, tem-se: } \\
& {\left[\begin{array}{c}
\mathrm{x}_{\mathrm{iu}}^{\mathrm{o}} \\
\mathrm{x}_{\mathrm{iu}}^{\mathrm{o}}
\end{array}\right]=\frac{1}{1,393695}\left[\begin{array}{cc}
1,453306 & 0,359458 \\
0,359458 & 1,047890
\end{array}\right]\left[\begin{array}{c}
2,506119 \\
1,992860
\end{array}\right]} \\
& {\left[\begin{array}{c}
\mathrm{x}_{\mathrm{iu}}^{\mathrm{o}} \\
\mathrm{x}_{2 \mathrm{u}}^{\mathrm{o}}
\end{array}\right]=\left[\begin{array}{c}
3,127401 \\
2,144760
\end{array}\right]}
\end{aligned}
$$$$
\text { Valores de } X_{1 u} \text { e } X_{2 u} \text { onde } \hat{Y}_{u} \text { atinge o ponto de máximo. }
$$ 
6. CONCLUSÕES

Com base nos resultados obtidos neste estudo, concluiu-se que:

6.1. Os valores de $\delta$ que tornam ortogonal o delineamento em ân gulos são dados por:

$$
\begin{aligned}
& \delta=\left(\frac{-24+\sqrt{1152-24 \mathrm{P}-6 \mathrm{P}^{2}}}{12-1,5 \mathrm{P}}\right)^{1 / 2} \text { se } 1<\dot{\mathrm{P}} 8 . \\
& \delta^{2}=5 / 3 \text { e portanto } \delta=1,290994 \text { se } \mathrm{P}=8 \\
& \delta=\left(\frac{24 \pm \sqrt{1152-24 \mathrm{P}-6 \mathrm{P}^{2}}}{1,5 \mathrm{P}-12}\right) \text { se } 8<\mathrm{P} \leqq 12
\end{aligned}
$$

6.2. Quando o delineamento em ângulos é ortogonal, usando-se um ou dois pontos centrais, os quatro pontos extremos são os correspondentes ao Fatorial $2 \times 2$ no qual os niveis estão codificados em -1 e +1 - $^{-}$ 
6.3. Existem dois valores para $\delta$ que tornam ortogonal o delineamento em àngulos, para cada valor de $P$, quando $9 \leqq P$ $\leq 11$.

6.4. Os valores das estimativas das variāncias das estimativas dos coeficientes do polinomio do segundo grau, quando o delineamento é ortogonal, são dados pelas expressões:

$$
\begin{aligned}
& \hat{V}\left(\hat{\beta}_{i}\right)=\frac{1}{r\left(4+6 \delta^{2}\right)} \cdot \hat{\sigma}^{2}, \quad \vec{V}\left(\hat{\beta}_{12}\right)=\frac{1}{r\left(4+1,5 \delta^{4}\right)} \cdot \hat{\sigma}^{2} \\
& \hat{V}\left(\hat{\beta}_{i i}\right)=\frac{1}{3 r \delta^{4}} \cdot \hat{\sigma}^{2}
\end{aligned}
$$

6.5. As estimativas dos coeficientes do polinómio do segundo grau, quando o delineamento é ortogonal, são dadas por:

$$
\begin{aligned}
& \hat{\beta}_{i}=\frac{\sum_{x_{i u} y_{u}}}{r\left(4+6 \delta^{2}\right)} \quad \hat{\beta}_{i i}=\frac{\sum_{u} x_{i u}^{2} y_{u}-\frac{\left(4+6 \delta^{2}\right)}{16+P} \sum_{u} y_{u}}{3 r \delta^{4}} \\
& \hat{\beta}_{12}=\frac{\sum_{u} x_{1 u} x_{2 u} y_{u}}{r\left(4+1,5 \delta^{4}\right)} \quad \hat{\beta}_{o}=\bar{Y}_{u}-\frac{4+6 \delta^{2}}{16+P}-\left(\hat{\beta}_{11}+\hat{\beta}_{22}\right)
\end{aligned}
$$

6.6. As somas dos quadrados referentes aos coeficientes, quando o delineamento é ortogonal, são dadas pelas expressões

$$
\operatorname{SQ}\left(\hat{\beta}_{i}\right)=\hat{\hat{\beta}}_{i} \cdot \sum_{\mathrm{u}} \cdot \mathrm{x}_{i \mathrm{u}} \mathrm{y}_{\mathrm{u}}
$$




$$
\begin{aligned}
& \operatorname{SQ}\left(\hat{\beta}_{i i}\right)=\hat{\beta}_{i i}\left[\sum_{u} x_{i u}^{2} y_{u}-\frac{\left(4+6 \delta^{2}\right)}{16+P} \sum_{u} y_{u}\right] \\
& S Q\left(\hat{\beta}_{12}\right)=\hat{\beta}_{12} \sum_{u} X_{1} x_{2 u} y_{u}
\end{aligned}
$$

6.7 - Na estimação de $\beta_{i}$ e $\beta_{i i}$, o Delineamento em Ângulos mostrou-se mais preciso do que o Fatorial $3^{2}$ e menos preciso do que os Fatoriais $5^{2}$ e $7^{2}$.

6.8 - Na estimação de $\beta_{12}$, o Delineamento em Ângulos é mais preciso do que o Fatorial $3^{2}$ quando são usados até quatro pontos centrais e menos preciso do que os Fatoriais $5^{2}$ e $7^{2}$.

6.9 - Levando-se em consideração a mesma ärea total a ser gasta, con clui-se que o Delineamento em Ângulos è mais preciso quando se usa um ponto central.

6.10 - Comparando-se o Delineamento em Ângulos com um ponto central com os Fatoriais de $3^{2}, 5^{2}$ e $7^{2}$, quando se utiliza a mesma ärea total do experimento, conclui-se:

6.9.1 - Quanto ao coeficiente $\hat{\beta}_{i}$, o delineamento em questão é tão preciso quanto o Fatorial $3^{2}$ e mais preciso do que os Fatoriais de $5^{2}$ e $7^{2}$.

6.9.2 - Quanto ao coeficiente $\hat{\beta}_{i i}$ o Delineamento em Ângulos com um ponto central é menos preciso do que os Fatoriais de $3^{2}, 5^{2}$ e $7^{2}$.

6.9.3 - Quanto ao coeficiente $\hat{\beta}_{12}$, o Delineamento em Ângulos com um ponto central é menos preciso do que o Fatorial $3^{2}$ e mais preciso do que os Fatoriais de $5^{2}$ e $7^{2}$. 


\section{REFERẼNCIAS BIBLIOGRĀFICAS}

BATISTA, L.B., 1976. Determinação de $\alpha$ para Tornar ortogonal o Delineamento Composto Centra1 (Box). Piracicaba. ESALQ/ /USP, 26 pp. (Dissertação de Mestrado).

BAtistA, L.B., 1978. Delineamento em Círculos. Pesq.Agropec. $\underline{\text { Bras. }}, \underline{13}(4): 9-15$.

BAtISTA, L.B. e S.C. e SILVA, 1978. Determinação de Fórmulas no Delineamento Composto Central (Box). Pesq. Agropec. $\underline{\text { Bras. }} \cdot \underline{13}(2): 39-47$.

BOX, G.E.P. e J.S. HUNTER, 1957. Multifactor Experimental Designs for Exploring Response Surface. Ann. Math. Stat, 28:195-241. 
BOX, G.E.P. e K.B. WILSON, 1951. On the Experimental Attainment of Optimum Conditions. Journal of the Royal statistical Society, série B, 13:1-45.

CAMPOS, H., 1967. Aspectos da Aplicação das Superfícies de Resposta a Ensaios Fatoriais $3^{3}$ de Adubação. Piracicaba. ESALQ/USP (Tese).

CONAGIM, A., 1979. Double Central Composite Designs. Resumos. da 10a. Conferência Internacional de Biometria, $3-4$, Guarujà, sP .

DANIEL, C., 1956. Fractional Replication in Industrial Research. Proc. In: JOHN, P.W.M., 1971. Statistical. Design and Analysis of Experiments. The Macmillan Co., New York, Cap. 8: 151-157。

FINNEY, D.J., 1945. The Fractional Replication of Factorial Arrangements. In: JOHN, P.W.M., 1971. Statistical Design, and Analysis of Experiments. The Macmillan Co., New York, Cap. 8:148-150. 
JORGE, J. de P.N., 1980. Delineamento Guadalupe para Três Fatores, Analis ado Através de Modelo de Regressão Polinomial Quadrätica. Piracicaba. ESALQ/USP, 56 pp. (Dissertação de Mestrado).

MARGOLIN, B.H., 1967. Systematic Methods of Analyzing $2^{m_{3}}$ Factorial Experiments with App1ications. In: JOHN, P.W.M., 1971. Statistical Design and Analysis of Experiments. The Macmi11an Co., New York, Cap. 8:158-160.

MORAES, R.S., 1969. Superfície Polinomial de Resposta Num Ensaio de Adubação com Níveis Não Equidistantes. Piracicaba. ESALQ/USP (Tese).

NELDER, J.A., 1963. Identification of Contrasts in Factorial Replication of $2^{\mathrm{n}}$ Experiments. In: JOHN, P.W.M., 1971 . Statistical Design and Analysis of Experiments. The Macmillan Co., New York, Cap. 8:158-160.

PENTEADO, A.F. e L.B. BATISTA, 1971. Eficiência do Ensaio Com posto Central (Box) em Comparação com os Fatoriais Completos de Dois Fatores. XIII Congresso Brasileiro de Ciência do So1o. Vitória, E.S. 
PIMENTEL GOMES, F., 1969. Novos Aspectos de Estudo Econômico de Ensaio de Adubação. Fertilité, 34:3-9.

PIMENTEL GOMES, F. e H. CAMPOS, 1972. The Efficiency of - Factorial $3^{3}$ Designs as Compared to a Central Composite Rotatable Designs. Potash Review, Fevereiro.

ROJAS, B.A., 1963. The San Cristóbal Designs for Fertilizer Experiments. In: JORGE, J. de P.N., 1980. Delineamento Guadalupe para Três Fatores, Analisadó Através de Modelo de Regressão Polinomial Quadrätica. Piracicaba. ESALQ / USP, 56 pp. (Dissertação de Mestrado).

ROJAS, B.A., 1972. The Orthogonalized San Cristóbal Design. In: JORGE, J. de P.N., 1980. Delineamento Guadalupe para Três Fatores, Analisado Através de Modelo de Regressão Poli nomial Quadrātica. Piracicaba. ESALQ/USP, 56 pp. (Disser tação de Mestrado).

SEARLE, S.R., 1971. Linear Mode1s. Nova York. John Wiley \& Sons. $532 \mathrm{p}$. 
STAVROU, J. e F.B. CADY, 1967. Counfounding the Triple Cube Response Surface Design to Reduce Block Size. Soil Sci.

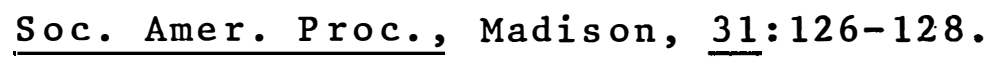

TRAMEL, T.E., 1957. A Suggested Procedure for Agronomic-economic Fertilizer Experiments. In: JORGE, J. de P.N., 1980.'Delineamento Guadalupe para Três Fatores, Analisado Através de Modelo de Regressão Polinomial Quadrática. Piracicaba. ESALQ / USP, 56 pp. (Dissertação de Mestrado).

VOSS, R. e J. PESEK, 1967. Yield of Corn Grain as Affected by Fertilizer Rates and Environmental Factors. Agron. J . Madis on, $\underline{59}: 567-572$.

YATES, F., 1937. The Design and Analysis of Factorial Experiments. Tech. Commun. Bur. Soil Sci. Harpenden, 35 : 77 pp. 